\title{
Small Business Innovation Research
}

\section{ABSTRACTS OF \\ PHASE II AWARDS}

2000

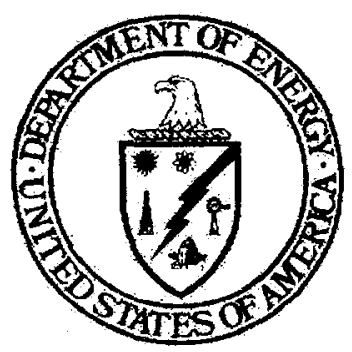

U.S. Department of Energy

Office of Science

SBIR Program

19901 Germantown Road

Germantown, MD 20874-1290 



\section{DISCLAIMER}

This report was prepared as an account of work sponsored by an agency of the United States Government. Neither the United States Government nor any agency thereof, nor any of their employees, make any warranty, express or implied, or assumes any legal liability or responsibility for the accuracy, completeness, or usefulness of any information, apparatus, product, or process disclosed, or represents that its use would not infringe privately owned rights. Reference herein to any specific commercial product, process, or service by trade name, trademark, manufacturer, or otherwise does not necessarily constitute or imply its endorsement, recommendation, or favoring by the United States Government or any agency thereof. The views and opinions of authors expressed herein do not necessarily state or reflect those of the United States Government or any agency thereof. 


\section{DISCLAIMER}

Portions of this document may be illegible in electronic image products. Images are produced from the best available original document. 


\section{PREFACE}

The SBIR program enables DOE to obtain effective, innovative solutions to important problems through the private sector, which has a commercial incentive to pursue the resulting technology and bring it to the marketplace. The growing number of awardees, many of them started in business in response to SBIR solicitations, is becoming a significant resource for the solution of high risk, high technology problems for the Department. As detailed below, this publication describes the technical efforts and commercialization possibilities for SBIR Phase II awards in Fiscal Year (FY) 2000. It is intended for the educated layman, and may be of particular interest to potential investors who wish to get in on the ground floor of exciting opportunities.

\section{The Federal SBIR Program}

This program, now completing its seventeenth year, was established in compliance with the Small Business Innovation Development Act of 1982, Public Law (PL) 97-219. The program was extended in October 1992 until October 2000 by the Small Business Research and Development Enhancement Act of 1992, PL 102564. Ten Federal agencies have SBIR programs - those with extramural research and development (R\&D) budgets of over $\$ 100$ million. The agencies were required to set aside $2.5 \%$ of their budgets in FY 2000 to fund SBIR projects. (This amounted to about $\$ 81$ million in FY 2000 for the Department of Energy.) Each agency solicits proposals from small science- and technology-based U.S. firms (with 500 employees or less) and makes awards competitively. SBIR research explores innovative concepts in important technological and scientific areas that can lead to valuable new technology and products. It supports innovative $R \& D$ and encourages conversion of that $R \& D$ into commercial applications of economic benefit to the Nation.

As prescribed in the legislation, the program is designed for implementation in three phases, with Phase I determining, insofar as possible, the scientific or technical merit and feasibility of ideas proposed for investigation. The period of performance in this initial phase is about six months, and the awards were limited to $\$ 100,000$ in FY 1999 . Phase II is the principal research or R\&D effort, and the awards were as high as $\$ 750,000$ in FY 2000 for work to be performedi a period of up to two years. In Phase III, commercial applications of the research or R\&D effort are pursued by the small businesses using non-Federal funding; alternatively, Phase III may involve follow-on non-SBIR Federal contracts for products or services desired by the Government. The program continues to strengthen the role that small, innovative firms play in meeting the Nation's research and developmentneeds and in commercializingideas developed through federally funded R\&D.

\section{Phase II Awards for FY 2000}

Contained in this booklet are abstracts of the Phase II awards made in FY 2000 under the DOE SBIR program.

DOE received 1135 grant applications in response to its annual solicitation, and on June 28, 1999, 202 were selected for Phase I funding. The Phase II SBIR program competition was limited to the 202 projects that subsequently proceeded to Phase I awards.

The deadline for the submission of Phase II applications was April 4, 2000. From the 184 applications submitted by this date, 91 were selected for Phase II awards. The listed amount of each award, in many instances, is subject to completion of award negotiations. 
DOE's FY 1999 annual Solicitation contained 40 technical topics in the areas of basic energy sciences, biological and environmental research, high energy and nuclear physics, fusion energy sciences, energy efficiency and renewable energy, fossil energy, nuclear energy, advanced scientific and computing research, environmental management, and nonproliferation and national security. Competition is keen because proposals must be of very high scientific and technical merit to be selected by one of the Department's program areas.

There are several measures of the program's success. Seventeen DOE SBIR projects have receivedR\&E100 Awards from Research and Development Magazine, which selects the 100 most significanttechnical products each year. Also, from the first ten cycles of awards (Phase II projects completed between 1986 and 1995), DOE SBIR winners have already received over $\$ 1$ billion in Phase III funding, triple the SBIR support they were given. This number is expected to grow significantly in the next few years.

Because so many small companies lack the business skills necessary for rapid commercializationof their technologies, DOE provides commercialization assistance through contractors that are competitively selected. In the Commercialization Assistance Program, successful Phase II awardees are given individual assistance in developing a business plan and in preparing presentations to potential investors. This program culminates in a series of presentations by the SBIR awardees to potential investors, including representatives from large corporations and venture capital firms. A second program provides Phase II awardees with TechnologyNiche Assessments, in which a DOE contractor identifies viable applications and strategic partners for the commercialization of SBIR technology. SBIR companies participating in these programs have already received more than $\$ 400$ million for the commercialization of their SBIR research. Sources of capital that wish to consider participation in future programs should contact Dr. Robert Berger; U.S. Department of Energy SBIR Program, SC-32, 19901 Germantown Road, Germantown, MD 20874-1290, (telephone 301-903-1414).

Robert E. Berger

SBIR Program Manager 


\section{TABLE OF CONTENTS}

\section{BIOMASS POWER}

1. Power from Farm Animals System Technology

Altex Technologies Corporation, Santa Clara, CA

2. The Development of a Modular System to Burn Farm Animal Waste to Generate Heat and Power

Spinheat Limited, Fairfield, CT

HIGH-TEMPERATURE ELECTRONICS FOR GEOTHERMAL DRILLING

3. High-Temperature Oscillator and Digital Clock

Linear Measurements, Inc., San Diego, CA 2

4. Capacitors for Extreme Temperature Applications

Sigma Technologies International, Inc., Tucson, AZ

5. A High Temperature MEMS Inclination Sensor for Geothermal Drilling

Silicon Designs, Inc., Issaquah, WA

\section{ADVANCED MEASUREMENT AND CONTROL TECHNOLOGIES FOR INDUSTRIAL MANUFACTURING APPLICATIONS}

6. Fiber Optic Sensor for Industrial Process Measurement and Control

MetroLaser, Inc., Irvine, CA

7. Real-time Gas Composition Analyzers for On-Line Process Control

Nanomaterials Research Corporation, Longmont, CO

8. Portable Parallel Beam X-Ray Diffraction System for In-Line Process Control in the Steel Industry

X-Ray Optical Systems, Inc., Albany, NY 5

\section{HYBRID ELECTRIC VEHICLE TECHNOLOGY}

9. High Efficiency Thermoelectric Power Conversion Devices

Hi-Z Technology, Inc., San Diego, CA

10. Improved Rotor Cooling for PM Machines

Unique Mobility, Inc., Golden, CO 


\section{SYSTEMS AND PRODUCTS FOR PASSIVE SOLAR LOW ENERGY BUILDINGS}

11. Passive Desiccant Air Conditioners

AIL Research, Inc., Princeton, NJ

12. SunGuard: A Roofing Tile for Natural Cooling

PowerLight Corporation, Berkeley, CA

\section{COAL/GAS POWER SYSTEMS}

13. Cost Saving Alternative for Stack Reformer Packaging in Tubular Solid Oxide Fuel Cells

Blasch Precision Ceramics, Inc., Albany, NY

14. Novel Backup Filter Device for Candle Filters

CeraMem Corporation, Waltham, MA

15. Advanced Catalytic Pilot for Advanced Gas Turbine Systems

Precision Combustion, Inc., New Haven, CT

16. Thermally Stable Catalysts for Methane Combustion

TDA Research, Inc., Wheat Ridge, CO

\section{RECOVERY AND UTILIZATION OF FOSSIL FUELS}

17. Improved Precursors for Oxygen-Selective Membranes in Practical Devices for Methane Conversion

CeraMem Corporation, Waltham, MA

18. Supported Flat Plate Thin Films for Oxygen Separation

Eltron Research, Inc., Boulder, CO 10

FUSION PLASMA SCIENCE RESEARCH

19. A Multi-Sensor Real-Time Resistive-Wall-Mode Identification Tool

FARTECH, Inc., San Diego, CA

\section{ENABLING TECHNOLOGIES FOR FUSION PLASMA EXPERIMENTS}

20. Power Supply for Plasma Heating

Diversified Technologies, Inc., Bedford, MA 
21. A New Radiation Resistant Epoxy Resin System for Liquid Impregnation Fabrication of Composite Insulation

Eltron Research, Inc., Boulder, CO

22. Advanced Heat Sink Materials for Fusion Energy Devices

Plasma Processes, Inc., Huntsville, AL

23. Fast Repetitive Arc Free Current Limiting Circuit Breaker

UTRON, Inc., Manassas, VA

\section{ADVANCED TECHNOLOGIES AND MATERIALS FOR FUTURE FUSION SYSTEMS}

24. Hybrid Three-Dimensional $\mathrm{SiC} / \mathrm{C}$ High Thermal Conductivity Composites

MER Corporation, Tucson, AZ 14

\section{ADVANCED CONCEPTS AND TECHNOLOGY FOR HIGH ENERGY ACCELERATORS}

25. Laser-Driven Cyclotron Autoresonance Accelerator - LACARA Omega-P, Inc., New Haven, CT

26. Multi-Terawatt, 50fs Laser for Laser Accelerator Applications

Positive Light, Inc., Los Gatos, CA 15

27. High-Performance Three-Dimensional Simulation Code for Laser Plasma Accelerators and Plasma Processing of Integrated Circuits, with a Graphical User Interface

Tech-X Corporation, Boulder, $\mathrm{CO}$ 16

\section{RADIO FREQUENCY ACCELERATOR TECHNOLOGY FOR HIGH ENERGY ACCELERATORS AND COLLIDERS}

28. Design of a $10 \mathrm{MW}, 91 \mathrm{GHz}$ Gyroklystron for Linear Accelerators

Calabazas Creek Research, Saratoga, CA

29. Low Field Solenoid Periodic Focusing of Accelerator Klystrons

California Tube Laboratory, Inc., Watsonville, CA

30. New Concepts for Pulsed Power Modulators

Diversified Technologies, Inc., Bedford, MA 18 


\section{HIGH-FIELD SUPERCONDUCTOR AND SUPERCONDUCTING MAGNET TECHNOLOGIES FOR HIGH ENERGY PARTICLES COLLIDERS}

31. Co-Processed Ceramic Insulation for High Field Accelerator Magnets

Composite Technology Development, Inc., Lafayette, CO.

32. Low-Cost, Long-Length Manufacturing of Continuously Melt-Quenched Processed, Full Stabilized, Multifilamentary High-Field $\mathrm{Nb}_{3}$ AL Superconductor

Plastronic, Inc., Subsidiary of Eurus Technologies Inc.,

Tallahassee, FL

33. Improvement of High Field Performance and Reliability of $\mathrm{Nb}_{3} \mathrm{Sn}$ Conductor

by Powder-in-Tube (PIT) Method

Supercon, Inc., Shrewsbury, MA

34. High Performance $\mathrm{Nb}_{i} \mathrm{Sn}(\mathrm{Ta})$ by Tin Enrichment and Increased Filament Content Superconducting Systems, Inc., Waltham, MA

35. A High Current Density Very Low Cost Niobium Tin (Titanium) Doped Conductor Utilizing A Novel Internal Tin Process, with Separate Stabilizing Elements Scalable to Modern Niobium Titanium Production Economics

Supergenics, Sarasota, FL

TECHNOLOGIES FOR THE NEXT-GENERATION

ELECTRON-POSITRON LINEAR COLLIDER

36. Automated Diamond Turning Lathe for the Production of Copper Accelerator Cells

DAC Vision, Inc., Carpinteria, CA

37. High Power Switch

Diversified Technologies, Inc., Bedford, MA

38. Hybrid NLC Modulator

Diversified Technologies, Inc., Bedford, MA

39. Adiabatic Forming of Copper Accelerator Cells for the Next Generation Linear Collider

LMC, Inc., De Kalb, IL.

\section{HIGH ENERGY PHYSICS DETECTORS}

40. Low Cost Support Structures, with New Advanced Composite Materials Tailored for Ultra-Stable Particle Tracking Detectors

HYTEC, Inc., Los Alamos, NM 


\section{NUCLEAR PHYSICS INSTRUMENTATION AND TECHNIQUES}

41. SQUID Susceptometers for Read Out of Magnetic Microcalorimeters

Hypres, Inc., Elmsford, NY

42. Nuclear Data Evaluation Tools

Scientific Digital Visions, Inc., San Jose, CA 24

\section{NUCLEAR PHYSICS ACCELERATOR TECHNOLOGY}

43. Electromagnetically Forming a Seamless Niobium Radio Frequency (RF) Superconducting Cavity

Advanced Energy Systems, Inc., Medford, NY

44. Development of High Power RF Windows for Next-Generation Superconducting and

Normal Conducting Accelerators

Advanced Energy Systems, Inc., Medford, NY

45. Superconducting RF Photocathode Electron Gun

Advanced Energy Systems, Inc., Medford, NY

46. High Power RF Window and its Input Coupler Technology

AMAC International, Inc., Newport News, VA

47. A High Efficiency Electron Cyclotron Resonance Ion Source System for the Production of Radioactive Ion Beams

Berkeley Ion Equipment, Inc., Santa Clara, CA

48. Development of New Lossy Material for Cryogenic and Ambient Applications

Ceradyne, Inc., Costa Mesa, CA

49. A High Force Actuator for SRF Cavity Tuning

Energen, Inc., Billerica, MA. 28

\section{WASTE CHARACTERIZATION}

50. Next-Generation, Portable XRF System

Photon Imaging, Inc., Northridge, CA

51. An Advanced Avalanche-Photodiode Based Spectroscopic Radiation Monitor

Radiation Monitoring Devices, Inc., Watertown, MA 


\section{WASTE TREATMENT AND STABILIZATION}

52. Separation of Metal lons from Liquid Waste Streams

Applied Sciences, Inc., Cedarville, $\mathrm{OH}$

53. Enhanced Selective Leaching of Chromium from Radioactive Sludges

Lynntech, Inc., College Station, TX

\section{TECHNOLOGY FOR TREATMENT, MANAGEMENT, AND DISPOSITION OF URANIUM HEXAFLUORIDE}

54. Production of High Value Fluorine Gases for the Semiconductor Industry

Starmet Corporation, Concord, MA 31

\section{ADVANCED ENVIRONMENTAL MONITORING TECHNOLOGY}

55. Fiber Optic Grating Based Moisture Sensor Blue Road Research, Fairview, OR

\section{ATMOSPHERIC MEASUREMENT AND SAMPLING TECHNOLOGY}

56. Development of III-Nitride UV Detectors

Avyd Devices, Inc., Costa Mesa, CA

57. Mercury Cadmium Telluride ( $\mathrm{HgCdTe})$ Detectors for Near Infrared Applications

Avyd Devices, Inc., Costa Mesa, CA

58. Low Temperature, High Altitude Humidity Sensor

Nanomaterials Research Corporation, Longmont, $\mathrm{CO}$

59. A Multi-Holographic Filter with a Liquid Crystal Scanner for Surface Optical Reflectance

Physical Optics Corporation, Torrance, CA.

60. A Low-Cost Automated Radiosonde Launch System

Yankee Environmental Systems, Inc., Turners Falls, MA

\section{CARBON CYCLE MEASUREMENTS \\ OF THE ATMOSPHERE AND THE BIOSPHERE}

61. A Diode Laser Sensor for High Precision Measurement of Terrestrial $\mathrm{CO}_{2}$ Sources and Sinks

Physical Sciences, Inc., Andover, MA 35 


\section{MEDICAL APPLICATIONS}

62. Rapid Processing of Positioning Information for Hand-Held Gamma Camera

PEM Technologies, Inc., Bethesda, MD

63. Small Animal SPECT Camera for Quantitative Molecular Medicine

Photon Imaging, Inc., Northridge, CA 36

\section{GENOME, STRUCTURAL BIOLOGY, AND RELATED BIOTECHNOLOGIES}

64. A Visual Data-Flow Editor Capable of Integrating Data Analysis and Database Querying CyberConnect Corporation, Storrs, CT

65. Genome Analysis Tools and Information Services

Genome Informatics Corporation, Oak Ridge, TN

66. Versatile Liquid Crystal Tunable Interference Filter for Chromosome Analysis

Physical Optics Corporation, Torrance, CA

\section{METAL FORMING}

67. A Generic Approach to Improved Semi-Solid Forming of Metals

Chesapeake Composites Corporation, New Castle, DE

68. High-Strain-Rate Superplastic Forging of Aluminum Alloys

Materials Modification, Inc., Fairfax, VA

\section{PROCESSING FOR SURFACE HARDNESS}

69. Functionally Graded, Nanocrystalline, Multiphase, Boron- and Carbon-Based Superhard Coatings

Spire Corporation, Bedford, MA 40

70. Large Area Filtered Arc Deposition of Carbon and Boron Based Hard Coatings

UES, Inc., Dayton, OH 40

\section{MATERIALS JOINING}

71. Hermetic Reaction Bonded Silicon Carbide Tube Joints with Microwave Heating

FM Technologies, Inc., Fairfax, VA 41 


\section{SUPERCONDUCTIVITY MATERIALS FOR ELECTRIC POWER}

72. Meter Length YBCO Coated Conductor Development

American Superconductor Corporation, Westborough, MA 42

NEUTRON INSTRUMENTATION

73. An Advanced Cold Moderator Using Solid Methane Pellets

Cryogenic Applications F, Inc., Clinton, TN

74. Three-Dimensional Si Imaging Array for Cold Neutrons

IntraSpec, Inc., Oak Ridge, TN 43

75. A High-Pressure ${ }^{3} \mathrm{He}$ Neutron Spin Filter

Science Research Laboratory, Inc, Somerville, MA 44

\section{ALTERNATIVE SYNTHETIC PATHWAYS FOR ENERGY EFFICIENT} AND ECONOMIC INDUSTRIAL CHEMICAL MANUFACTURE

76. Novel Catalyst for $\mathrm{CH}_{4}-\mathrm{CO}$ Conversion

CeraMem Corporation, Waltham, MA 44

\section{REACTIVE SEPARATIONS}

77. Membrane Reactor Designs for the Production of Olefins

Membrane Technology and Research, Inc., Menlo Park, CA

78. Sonic Assisted Membrane Processing

Montec Associates, Inc., Butte, MT

79. Economic and Self-Sustaining Production of Saleable Products from Waste Anions Using Phase-Transfer Catalysis

PTC Value Recovery, Mt. Laurel, NJ 46

INNOVATIVE R\&D FOR ADVANCED, LOW-EMISSION, ELECTRIC AND HYBRID VEHICLE TECHNOLOGIES

80. Flame Retardant Electrolytes for Li-Ion Batteries

EIC Laboratories, Inc., Norwood, MA

81. Nonflammable Lithium-Ion Battery Electrolytes

TechDrive, Inc., Oakbrook Terrace, IL 


\section{COMPUTATIONAL GEOSCIENCES}

82. A Geometry-Based Interactive Pre- and Post-Processor for Reservoir Simulation and Visualization

Thunderhead Engineering Consultant, Inc. (Joint Venture), Manhattan, KS 48

\section{HIGH PERFORMANCE NETWORKS AND APPLICATIONS}

83. Al, An ESNET Differentiated Services Allocation System

Aldea Communication, Inc., Carlsbad, CA

84. Holographic Memory Module with Ultra-High Capacity and Throughput

MetroLaser, Inc., Irvine, CA

85. Plug-and-Play Phase Conjugate Quantum Cryptography for Secure Private and

Public Key Distribution

Physical Optics Corporation, Torrance, CA 50

86. Low Cost $10 \mathrm{~Gb} / \mathrm{s}$ Optical Links for Gigabit Networks

Princeton Electronic Systems, Inc., Princeton, NJ 50

\section{ADVANCED SENSORS AND DATA ANALYSIS TECHNIQUES FOR NATIONAL SECURITY APPLICATIONS}

87. Chemosensor Array for Detecting the Proliferation of Weapons of Mass Destruction Intelligent Optical Systems, Inc., Torrance, CA

88. A New Microfluidic System for Detection of Bacterial Agents on Contaminated Surfaces

Lynntech, Inc., College Station, TX

89. Compact Reflector Telescope

Optra, Inc., Topsfield, MA

90. Fast Analysis of Ultraspectral Measurements of Chemical Plumes

Spectral Sciences, Inc., Burlington, MA 52

\section{ADVANCED RESEARCH IN SUPPORT OF THE COMPREHENSIVE NUCLEAR-TEST BAN TREATY}

91. Seismic Monitoring System Calibration Using Ground Truth Database

Multimax, Inc., Largo, MD 


\section{BIOMASS POWER}

1

Power from Farm Animals System Technology--Altex Technologies Corporation, 650 Nuttman Street, Suite 114, Santa Clara, CA 95054-2620; 408-982-2303

Dr. John T. Kelly, Principal Investigator

Dr. Mehdi Namazian, Business Official

DOE Grant No. DE-FG03-99ER82738

Amount: \$712,084

Animal wastes are an environmental problem, particularly in feed lot areas where animals are concentrated. If the wastes could be upgraded to a fuel and burned in efficient small-scale engines, high value electric power could be produced, while mitigating environmental problems. This project will develop a small-scale power generation method to pretreat and combust the animal waste, so that reliable and low cost electric power can be generated using existing engines. Preliminary economic analyses have shown that the concept will be competitive in geographical areas of interest. In Phase I, opportunities for power generation from animal waste were identified and concept designs were prepared to address those opportunities. In addition, available test equipment was utilized to fill in key data gaps. Test results were then used to update the concept design and, with the support of manufacturers, an economic analysis was performed to show feasibility: In Phase II, a proof-of-concept system will be constructed and installed at an operating farm where the system will be tested. Test results will then be combined with economic analysis to evaluate the viability of the concept for commercial applications.

Commercial Applications and Other Benefits as described by the awardee: The concept should allow for the generation of electric power from animal wastes, primarily in remote rural areas in the U.S. and abroad. Potentially over 34 million tons of animal wastes could be beneficially used, thereby reducing surface and ground water environmental problems. Also the burning of this renewable waste could reduce $\mathrm{CO}_{2}$ greenhouse gas emissions by over 30 million tons.

2

The Development of a Modular System to Burn Farm Animal Waste to Generate Heat and Power--Spinheat Limited, 1222 Bronson Road, Fairfield, CT 06430-2824; 203-2596101

Mr. Michael J. Virr, Principal Investigator

Mr. Michael J. Virr, Business Official

DOE Grant No. DE-FG02-99ER82890

Amount: $\$ 749,921$

This project will develop a range of modular systems to economically convert farm animal wastes to heat and electric power using a new packaged fluid bed boiler and micro-turbine. The Internally Circulating Fluid Bed (ICFB) boiler minimizes emissions and ensures maximum efficiency, thus eliminating the dumping of animal wastes on the ground and the attendant chance of contaminating ground water. The complete system will be incorporated into a small co-generation plant that will utilize distributive control for unattended operation. Phase I focused on the design of an integrated system. Preliminary fuel tests in a fluid bed were conducted to establish material and energy balances. A preliminary market and resource assessment was performed, along with initial estimates of plant costs and environmental emissions/benefits. In Phase II, a suitable site will be established, and a detailed design of a 150 $\mathrm{kW}$ plant will be completed. This will be followed by the erection and commission of a 150 $\mathrm{kW}$ demonstration plant for burning poultry litter. 
Commercial Applications and Other Benefits as described by the awardee: Packaged cogeneration plants, including ICFB boilers and micro-turbines, should be sold to farmers, poultry producers, and similar industry waste generators, to save heat power costs.

\section{HIGH-TEMPERATURE ELECTRONICS FOR GEOTHERMAL DRILLING}

3

\author{
High-Temperature Oscillator and Digital \\ Clock--Linear Measurements, Inc., 4174 Sorento \\ Valley Boulevard, San Diego, CA 92121-1483; \\ 619-535-2172 \\ Mr. Harper J. Whitehouse, Principal Investigator \\ Mr. Robert Hatch, Business Official \\ DOE Grant No. DE-FG03-99ER82812 \\ Amount: \$747,235
}

The Department of Energy and the renewable energy industry need electronic devices and sensors to control and optimize the drilling of geothermal wells. This project will develop a high-temperature oscillator and digital clock to provide the precision timing required to control other components and subsystems in such high temperature systems as measurement-whiledrilling (MWD), logging-while-drilling (LWD), and data telemetry. The high-temperature oscillator and digital clock will utilize a surface acoustic wave (SAW) device in which a pair of metal transducers operates on a piezoelectric crystal substrate. An electronic amplifier connected to one transducer generates an acoustic surface wave that propagates along the surface to the second transducer where the acoustic wave is converted into an electrical signal and fed back to first transducer. The analog electrical signal from the surface acoustic wave oscillator will be electronically converted to a digital clock signal. In Phase I, the new quartz-like material langasite (LGS, $\mathrm{La}_{3} \mathrm{Ga}_{5} \mathrm{SiO}_{14}$ ) was shown to have a SAW crystal cut with a zero temperature coefficient of frequency at $160^{\circ} \mathrm{C}$. For the high-temperature electronics, specially fabricated gallium arsenide (GaAs) transistors were shown to operate at $400^{\circ} \mathrm{C}$. High-temperature circuit board materials were identified along with the other necessary passive electronic components needed. In Phase II all of the acoustic and electronic components will be combined in an experimental prototype high-temperature oscillator and digital clock.

Special surface mount circuit board technology will be used to minimize thermally-induced stresses. The prototype circuit will be tested at temperatures from near $0^{\circ} \mathrm{C}$ to temperatures greater than $300^{\circ} \mathrm{C}$, and the components will be optimized to maintain a frequency stability of $+1-$ 500 parts per million (ppm) accuracy over a temperature range from $20^{\circ} \mathrm{C}$ to greater than $300^{\circ} \mathrm{C}$.

Commercial Applications and Other Benefits as described by the awardee: In addition to geothermal well drilling and logging, the hightemperature digital clock should be useful wherever temperature and pressure measurements are needed in high-temperature, adverse environments. Applications include aircraft piston, jet engine, and automobile engine control, as well as nuclear power plant safety.

4

Capacitors for Extreme Temperature Applications--Sigma Technologies International, Inc., 10960 N. Stallard Place, Tucson, AZ 85737; 520-575-8013

Dr. Angelo Yializis, Principal Investigator

Dr. Angelo Yializis, Business Official

DOE Grant No. DE-FG03-99ER82884

Amount: \$750,000

There is a need to develop high temperature electronic components that can withstand the harsh environment of geothermal well drilling. The development of state-of-the-art polymer/ metal capacitors would introduce major 
improvements to high temperature scalable, onthe-spot, information systems associated with well drilling, data telemetry, and process control. This project will use high-rate vacuum-based polymer/metal multilayer (PML) technology to fabricate solvent free, low cost, lightweight, high temperature capacitors. Phase I successfully demonstrated that the polymer multilayer capacitors, composed of thousands of high temperature polymer dielectric layers separated with self-healing metal electrodes, have a superior performance over a wide range of temperatures $\left(-235^{\circ} \mathrm{C}\right.$ to $\left.260^{\circ} \mathrm{C}\right)$. The upper temperature limit was extended by eliminating the low melting point solder at the capacitor's termination through the use of an arc metal spray method and by improving the polymer dielectrics used. In Phase II, PML capacitors with different polymer dielectrics will be further optimized and evaluated under several application-based conditions. Long-term life tests will be performed, and the temperature and performance limits will be investigated.

Commercial Applications and Other Benefits as described by the awardee: The PML capacitors should find uses in the high temperature capacitor and sensor market sectors, including deep geothermal wells, power electronic applications for aircraft, automotive, and specialty instrumentation.

\section{5}

A High Temperature Microelectro-mechanical Inclination Sensor for Geothermal Drilling-Silicon Designs, Inc., 1445 NW Mall Street, Issaquah, WA 98027; 425-391-8329

Mr. John C. Cole, Principal Investigator

Mr. John C. Cole, Business Official

DOE Grant No. DE-FG03-99ER82885

Amount: $\$ 749,224$

Directional drilling, commonly used in oil and gas production, uses magnetometers and inclination sensors to measure the azimuth and inclination of the drill bit. However, for geothermal wells, the drilling equipment must be capable of operating at much higher temperatures than for oil and gas. No miniature inclination sensor is now available to accurately measure the direction of earth gravity at such temperatures.

This project will develop a family of high temperature accelerometers for measuring inclination and vibration at operating temperatures up to $300^{\circ} \mathrm{C}$. With these accelerometers and other digital components, temperature-compensated inclination sensor units will be built for geothermal drilling. Phase I consisted of developing a preliminary design for a high temperature integrated circuit (IC), evaluating the performance of accelerometer sensing elements at high temperature, identifying suitable packaging methods, and designing a high temperature test system. Phase II will consist of developing the high temperature IC and building the necessary equipment for calibrating and testing the high temperature accelerometers.

Single and three axis accelerometers will then be assembled and tested.

Commercial Applications and Other Benefits as described by the awardee: The high temperature accelerometers and inclination sensors would be available to producers of directional drilling equipment for geothermal and oil and gas drilling. They also should be useful in other high temperature industrial applications, as well as in space and military applications where high radiation dose rates are present. 


\section{ADVANCED MEASUREMENT AND CONTROL TECHNOLOGIES FOR INDUSTRIAL MANUFACTURING APPLICATIONS}

6

Fiber Optic Sensor for Industrial Process Measurement and Control--MetroLaser, Inc., 18010 Skypark Circle, Suite 100, Irvine, CA 92614-6428; 949-553-0688

Dr. Peter A. DeBarber, Principal Investigator

Dr. Cecil F. Hess, Business Official

DOE Grant No. DE-FG03-99ER82828

Amount: $\$ 749,966$

An increasingly competitive global marketplace requires energy- and waste-intensive industries to improve efficiency while also generating fewer pollutants and other wastes. Achieving the necessary improvements requires improved inprocess chemical sensor technology that will provide faster and more accurate measurements of key parameters. Existing monitoring instrumentation is either difficult to use, expensive, or has insufficient time response. This project will develop advanced diode laser-based fiber optic sensors for direct, rapid, and quantitative measurement of gaseous species and temperature in industrial processes. In Phase I, detailed computer simulations were run for various industrial applications. Spectral absorption characteristics that show the greatest promise were identified. A laboratory breadboard system was designed and built, and feasibility experiments were carried out which demonstrated the quantitative capabilities of the approach. In Phase II, a robust prototype will be designed and built for a selected DOE Industry-of-the-Future application. This will be tested in a laboratory environment and then demonstrated at a DOE-approved site.

Commercial Applications and Other Benefits as described by the awardee: A compact, rugged, and inexpensive fiber optic chemical sensors for real-time in situ measurements of gas temperature and chemicals will be attractive in industrial applications where conventional monitoring systems are unsatisfactory (e.g., too large, expensive, slow or not versatile enough).

7

Real-time Gas Composition Analyzers for On-Line Process Control--Nanomaterials Research Corporation, 2620 Trade Center Avenue, Longmont, CO 80503-7551; 303-702-1672

Dr. Stephen S. Williams, Principal Investigator

Dr. Tapesh Yadav, Business Official

DOE Grant No. DE-FG03-99ER82843

Amount: $\$ 601,398$

The optimization of combustion efficiency and the minimization of exhaust emissions are required for the proper operation of virtually every industrial process that burns fuel. To accomplish this, sensors for process control are needed that can detect changes in the air-to-fuel ratio in a proportional manner (compared to the non-linear sensors that are currently used) during the combustion process. This project will develop and commercialize a novel, solid-state sensor that offers the desired proportional response for controlling combustion processes in materials processing, power generation, and waste incineration. Phase I demonstrated the feasibility of using a new sensor material to produce advanced solid-state combustion efficiency sensors that will effectively monitor the air-to-fuel (A/F) ratio in pre-and postcombustion streams. The sensors had rapid response at high temperatures and at various oxygen concentrations, and, most importantly, the desired proportional response was achieved. An alternative fabrication technique enabled sensor miniaturization, and sensor-to-sensor reproducibility was increased. Phase II will develop packaged sensors for use in process control applications. Further refinements will be made in the fabrication technique. Other 
challenges include developing an internal heater for the sensor elements, packaging the sensor elements, and developing the electronics for the packaged sensor.

Commercial Applications and Other Benefits as described by the awardee: These air-to-fuel ratio sensors for process control should find use in at the combustion industry to conserve energy to increase product yield and to reduce pollution and waste. Specific applications for the sensors include: flue-gas/exhaust monitoring, materials processing, and open flame monitoring.

\section{8}

Portable Parallel Beam X-Ray Diffraction System for In-Line Process Control in the Steel Industry--X-Ray Optical Systems, Inc., 30 Corporate Circle, Albany, NY 12203: 518-464-3334

Dr. Michael Haller, Principal Investigator

Mr. David Usher, Business Official

DOE Grant No. DE-FG02-99ER82918

Amount: \$749,831

In-line process control is a vital method for improving and assuring product quality, maximizing process efficiency, and minimizing waste generation in many manufacturing industries, including the steel industry. X-ray diffraction, one of the most versatile analytical tools available, could provide valuable contributions. However, its use has essentially been confined to the laboratory environment because current techniques require prepared samples and the equipment is not suitable for use in a harsh manufacturing environment. This project will develop a parallel beam, $x$-ray diffractometer for in-line monitoring of phase, grain size, grain orientation, and residual stress in steel sheets. No sample preparation will be required, and the data quality will not be affected by sample vibration or roughness. Polycapillary $\mathrm{x}$-ray optics will collect $\mathrm{x}$-rays over a large solid angle from a low-power $x$-ray source and form an intense large area parallel beam. In Phase I, galvanized annealed steel samples were measured on a parallel-beam diffractometer. A concept was developed for more versatile parallel-beam diffractometer that can be placed within the production line and allow uninterrupted measurement. This will be constructed in Phase II. Also, polycapillary optics will be designed and implemented into the diffractometer. Once built, the complete system will be incorporated into a pilot line of a major U.S. steel manufacturer.

Commercial Applications and Other Benefits as described by the awardee: Currently x-ray diffraction is widely used off-line to provide critical feedback to manufacturing. If successful, continuous in-line monitoring of material properties using $\mathrm{x}$-ray diffraction should improve yield, decrease power requirements, and improve quality for a wide range of industries including steel, aluminum, cement, and pharmaceuticals.

\section{HYBRID ELECTRIC VEHICLE TECHNOLOGY}

9

\author{
High Efficiency Thermoelectric Power \\ Conversion Devices--Hi-Z Technology, Inc., \\ 7606 Miramar Road, Suite 7400, San Diego, CA \\ 92126-4202; 619-695-6660 \\ Dr. Saeid Ghamaty, Principal Investigator \\ Mr. Norbert Elsner, Business Official
}

\section{DOE Grant No. DE-FG03-99ER82797}

Amount: $\$ 750,000$

Quantum well films have the potential to more than double the efficiency of thermoelectric devices used to convert waste heat into electricity, Quantum well films consist of many very thin alternating ceramic layers, each approximately $100 \AA$ thick, deposited on a suitable substrate (typically $\mathrm{Si}$ ). Unfortunately, 
the substrate, which is needed for mechanical support, can act as a thermal short. This can be overcome by maximizing the ratio of film thickness to substrate thickness. This project will fabricate very thick $(>10 \mu)$ quantum well films on a very thin Si substrate $(\sim 5 \mu)$ leading to very efficient and stable power generation devices. Phase I demonstrated outstanding performance of multilayer boron carbine films for thermoelectric energy conversion. Individual films were found to have a figure-of-merit that was on order of magnitude greater than the bulk material. Combinations of these multilayer boron carbide materials were paired with multilayer $\mathrm{Si} / \mathrm{SiGe}$ or bulk bismuth telluride, and a conversion efficiency of up to 24 percent was measured. The main focus in Phase II will be on increasing the ratio of multilayer film to substrate, by a progressive approach to thicker films and thinner substrates. Deposition and annealing process conditions will be selected to allow this increase without the build-up of unacceptable stress. Device structures will be built and tested.

Commercial Applications and Other Benefits as described by the awardee: The technology should find application as waste heat recovery devices from internal combustion engines in trucks and hybrid cars and from combustion areas at power stations and oil refineries. Other applications could include "self powered" boilers providing their own electrical power for control and small-scale but highly efficient power sources for MEMs.

Improved Rotor Cooling for PM Machines-Unique Mobility, Inc., 425 Corporate Circle, Golden, CO 80401-5635; 303-278-2002

Mr. Jon F. Lutz, Principal Investigator

Mr. Donald A. French, Business Official

DOE Grant No. DE-FG03-99ER82914

Amount: \$749,072

Electric drive motors for hybrid electric vehicles require improvements in cost reduction and power density. In particular, the brushless permanent 6 magnet (PM) motor, which has thus far demonstrated superior performance, requires improvements in both cost-effectiveness and packaging. This project will improve the rotor cooling of a brushless PM motor by utilizing an innovative self-cooling concept. This cooling method not only will reduce the cost of the permanent magnets, but also will reduce the size of the motor for a given power rating. Phase I produced several innovative cooling ideas that were fitted to a current motor. The motor was run through thermal tests to optimize the cooling effectiveness, cost effectiveness, and modularity. Phase II will design a modular set of three motors that will have will have higher power density and be more cost effective to produce than those using the current technology. Cost reductions will be achieved by lowering the magnet content in the motors (cooler rotors), sharing common parts between designs, and using advanced manufacturing techniques.

Commercial Application and Other Benefits as described by the awardee: The hardware developed as part of this program should have application to parallel hybrid, series hybrid, battery electric, and fuel cell electric passenger cars. Other applications include parallel hybrid sport utility vehicles and trucks, series hybrid wheel motors for large vehicles, and starter/motor/generators for high voltage and 42 volt vehicles. 


\section{SYSTEMS AND PRODUCTS FOR PASSIVE SOLAR LOW ENERGY BUILDING}

11

Passive Desiccant Air Conditioners--AIL Research, Inc., P.O. Box 3662, 50 Washington Road, Princeton, NJ 08543-3662; 609-452-2950 Mr. Jeffrey A. Miller, Principal Investigator Dr. Andrew Lowenstein, Business Official DOE Grant No. DE-FG02-747650

Amount: \$747,650

In dry climates, such as those found in the southwest, evaporative coolers can maintain comfortable conditions in homes and commercial buildings while using much less energy than freon-based and other similar cooling systems. This passive cooling system passes air through wet media, with the evaporation of water cooling the air. Their performance decreases as humidity levels rise, hence their limited geographical use. This project will develop a passive desiccant air conditioner that will allow evaporative cooling to be used in more humid environments, thereby increasing its potential use with its attendant economic and environmental benefits. In Phase I, a flexible computer model of the passive desiccant air conditioner was developed. The model was used to design a product that used much less energy than a conventional air conditioner over a cooling season while having acceptable manufacturing costs. In Phase II, the design of the passive desiccant air conditioner will be refined through working with a manufacturer; a subscale model of a commercial system will be demonstrated on a building; manufacturing costs will be determined; and potential installation, operation, and maintenance problems will be identified and corrected.

Commercial Applications and Other Benefits as described by the awardee: A passive desiccant cooling system should save energy when applied to both residences and commercial buildings. It can also be used to reduce energy use in many industrial processes that now use cooling towers. Such energy savings will reduce emissions of carbon dioxide and pollutants, conserve fossil fuels, and reduce the need for additional power plants, especially those built for peak power needs.

12

SunGuard: A Roofing Tile for Natural Cooling--PowerLight Corporation, 2954 San Pablo Avenue, Berkeley, CA 94710; 510-540-0550

Mr. Thomas L. Dinwoodie, Principal Investigator

Mr. Thomas L. Dinwoodie, Business Official

DOE Grant No. DE-FG03-99ER82862

Amount: $\$ 749,899$

Maintaining a cool roof deck can significantly reduce air conditioning costs in coolingdominated climates while reducing humidity build-up and other moisture problems within the home. A preferred method of cooling roof decks is by natural convection, which should have wide market acceptance for residential applications compared with reflective or other methods. This project will develop novel residential roofing technology that accomplishes significant roof deck temperature reduction through strictly passive means. The technology integrates cooling load avoidance and humidity removal to reduce building cooling loads. Phase I optimized product construction and validated product performance under full-scale system testing. A system prototype was constructed for independent testing and validation at a national laboratory. The prototype reduced heat flow through the roof on a typical summer day by 90 percent. In Phase II, design enhancements will be developed and incorporated. These include a roof mounting system to facilitate installation, improved material and processing specifications to reduce fabrication costs, and enhanced convection for improved thermal performance. Critical product certifications and commercial partnerships will be obtained. 
Commercial Applications and Other Benefits as described by the awardee: The passive cooling technology targets residential roofing for roof slopes of 2:12 and higher, representing 77 percent of the residential roofing market. With just 5 percent market penetration, the nation would save over 35 million barrels of oil per year, with annual environmental savings of 18.6 million tons of carbon dioxide, 80 kilotons of nitrogen oxides, and 178 kilotons of sulfur oxides.

\section{COAL/GAS POWER SYSTEMS}

\section{3}

Cost Saving Alternative for Stack Reformer Packaging in Tubular Solid Oxide Fuel Cells-Blasch Precision Ceramics, Inc., 580 Broadway, Albany, NY 12204-2802; 518-436-1263

Mr. David A. Larsen, Principal Investigator

Mr. David W. Bobrek, Business Official

DOE Grant No. DE-FG02-99ER82752

Amount: $\$ 749,855$

Solid oxide fuel cells are a very efficient and clean method of power generation, but to be commercially viable, significant cost reduction is necessary to reduce the cost of power generated. The stack reformer packaging in tubular solid oxide fuel cells is expensive because it is comprised of machined high alumna ceramic fiber board, costing over $\$ 88,000$ for a 100 kilowatt unit. This project will replace the current machined fiberboard with a much more economical ceramic particulate material, and develop near-net-shape ceramic technology to form the new ceramic into the appropriate shapes. Phase I developed a fine-grained ceramic particulate-based material that was then formed with a unique process into an economical prototype outer-stack reformer-board shape for tubular solid oxide fuel cells. Over 40 ceramic material compositions were formulated and characterized, to achieve targeted properties including no detectable crystalline silica content and reasonable strength. In Phase II, the ceramic composition data and small scale process techniques will be used as the basis for ceramic material optimization and prototype production of full size, large, complex ceramic shapes for use in tubular solid oxide fuel cell packages. The 8 structural refractory design of the entire fuel cell package will also be reviewed by a major fuel cell manufacturer, followed by design of near-net shape ceramic components for optimum economy in shape manufacture and package assembly, without sacrificing cell performance.

Commercial Applications and Other Benefits as described by the awardee: The technology should have application in tubular solid oxide fuel cells where significant savings in the cost of currently-used, machined ceramic fiber boards should be achieved. Savings of at least $\$ 55,000$ (65 percent) per 100 kilowatt fuel cell unit should be attained with ceramic particulate-based shapes, resulting in a reduction in the cost of power generated.

Novel Backup Filter Device for Candle FiltersCeraMem Corporation, 12 Clematis Avenue, Waltham, MA 02453; 781-899-4495 Dr. Bruce A. Bishop, Principal Investigator Mr. Robert L. Goldsmith, Business Official DOE Grant No. DE-FG02-99ER82760

Amount: $\$ 750,000$

Hot gas particulate removal is required for advanced coal conversion process systems to protect downstream equipment such as turbines, catalyst beds, and fuel cells. The currently preferred means of particulate removal is filtration with candle filters. However, candle filters have not shown the reliability necessary to be commercially viable. This project will develop a simple backup filter for candle filters, which will protect downstream equipment in case of 
candle failure. In Phase I, prototype filters were fabricated and tested under ambient conditions to select preferred pore size and geometry. Filters with preferred characteristics were subjected to tests at $1500^{\circ} \mathrm{F}$ and $150 \mathrm{psig}$ and showed acceptable flow-through characteristics and rapid plugging on candle failure. In Phase II, full size filters will be developed with pore sizes tailored to meet particle retention needs for various process requirements for protection of downstream equipment. Filters will be tested more extensively under both ambient conditions for process performance at full scale and in hot gas tests in a lengthy (500-hr) life test.

Commercial Applications and Other Benefits as described by the awardee: The fail-safe filter being developed would be used in advanced coal conversion processes to protect downstream equipment in case of primary filter failure. Such protection is required for these processes to have a reliability factor acceptable to the utility industry and funding sources. Without such protection, advanced coal conversion processes will have limited acceptance.

\section{5}

Advanced Catalytic Pilot for Advanced Gas Turbine Systems--Precision Combustion, Inc., 25 Science Park, \#24, New Haven, CT 06581$1968 ; 203-787-8614$

Dr. Shah Etemad, Principal Investigator

Mr. Paul M. Donahe, Business Official

DOE Grant No. DE-FG02-99ER82863

Amount: $\$ 750,000$

Current natural gas turbine engine systems operate with reduced efficiencies because aftertreatments are required to reduce $\mathrm{NO}_{\mathrm{x}}$ emissions. By enhancing Dry Low $\mathrm{NO}_{x}$ (DLN) gas turbines with catalytic pilot technology, $\mathrm{NO}_{\mathrm{s}}$ emissions could be reduced below 9 ppm, lower than is feasible with current DLN systems using standard non-catalytic pilots. This project will develop a catalytically stabilized lean premixed fuel/air pilot burner to replace the standard diffusion flame pilot burner. All pilot burner functions will be performed while achieving low
NO $\mathrm{x}_{\mathrm{x}}$ emissions (through lean premixed operation) and lean stability (through the catalytic reaction of a portion of the fuel mixture). In Phase I, computational fluid dynamics and stress analysis calculations were performed, and catalytic pilot prototypes were developed for each of two engine manufacturer's machines. One of these was durably tested for twelve hours in one of the engine manufacturer's combustors at 8 and 11 atmosphere's pressure, stably achieving $7 \mathrm{ppm}$ $\mathrm{NO}_{\mathrm{s}}$ in a DLN combustor design that previously achieved only 18-20 ppm NO . In Phase II, the catalytic pilot technology will be integrated with specific engine applications. Full scale, full pressure test opportunities will be provided by two major manufacturers of gas turbines.

Commercial Applications and Other Benefits as described by the awardee: This technology should enable the lowering of $\mathrm{NO}_{\mathrm{x}}$ emissions from DLN (dry low $\mathrm{NO}_{\mathbf{x}}$ ) combustion systems for natural gas fueled ground power ATS (Advanced Turbine Systems) gas turbine engines. A substantial retrofit market could also emerge for replacing conventional high $\mathrm{NO}_{\mathrm{x}}$ pilots with ultra-low $\mathrm{NO}_{\mathrm{x}}$ catalytic pilots.

\section{6}

Thermally Stable Catalysts for Methane Combustion--TDA Research, Inc., 12345 West 52nd Avenue, Wheat Ridge, CO 80033-1917; 303-940-2301

Dr. David T. Wickham, Principal Investigator

Mr. Michael E. Karpuk, Business Official

DOE Grant No. DE-FG03-99ER82902

Amount: \$590,211

Catalytic combustion is a very promising technology for reducing $\mathrm{NO}_{\mathrm{x}}$ emissions from gas turbine power generators. Its primary difficulty is that the catalysts must be able to withstand extremely high temperatures, up to $1500^{\circ} \mathrm{C}$. Hexaaluminates are compounds that have potential to be suitable catalysts, but their activities are still too low and they are difficult to synthesize. A fundamentally different route has been developed for the production of high surface area hexaaluminate catalysts. This method 
would allow for the preparation of many compounds with well-controlled stoichiometries for testing. In Phase I, many combinations of bimetallic hexaaluminate catalysts were synthesized. Several catalyst formulations that have adequate activity and thermal stability for use in a gas turbine application were identified. One of the catalysts was tested in a wall-mounted configuration and found to be as active as it was in the powder form. Phase II will scale up the production of the combustion catalysts for demonstration on an operating gas turbine. Additional tests to optimize the catalyst formulation will be performed, and then pilot and full-scale testing of the optimized catalyst in monolithic form will be carried out. A full sized reactor will be fabricated, installed on a gas turbine generator, and tested under normal operating conditions.

Commercial Applications and Other Benefits as described by the awardee: The use of a combustion catalyst to control $\mathrm{NO}_{\mathrm{x}}$ will allow power generators to meet $\mathrm{NO}_{\mathrm{x}}$ emission standards without installing costly post-combustion treatment. This should result in significant capital and operational cost savings.

\section{RECOVERY AND UTILIZATION OF FOSSIL FUELS}

17

Improved Precursors for Oxygen-Selective Membranes in Practical Devices for Methane Conversion--CeraMem Corporation, 12 Clematis Avenue, Waltham, MA 02453; 781-899-4495

Dr. Richard Higgins, Principal Investigator

Dr. Robert L. Goldsmith, Business Official DOE Grant No. DE-FG02-99ER82761

Amount: $\$ 750,000$

Liquid transportation fuels derived from natural gas have great potential to supplement or replace fuels derived from imported crude oil. Converting natural gas to these fuels is not currently costeffective, but recent laboratory developments involving use of oxygen-permeating ceramic membrane reactors clearly show feasibility to convert natural gas economically. However, improved materials and device fabrication methods are required to scale up such reactors. This project will demonstrate a cost-effective method of synthesizing improved precursors for the oxygen-permeating materials used in state-ofthe-art ceramic membrane reactors for natural gas conversion. In Phase I, the feasibility of the synthesis of the precursor was demonstrated; the predominant focus was on the control of chemical composition. Preliminary work was completed in the area of membrane fabrication. In Phase II, the precursor synthesis method will be comprehensively investigated to obtain particulate precursors with optimal physical and chemical properties. Composite oxygenpermeating membranes will be fabricated using them, and their performance properties will be demonstrated. Alternative membrane fabrication methods also will be investigated.

Commercial Applications and Other Benefits as described by the awardee: The availability of these materials would facilitate the fabrication and scale-up of ceramic membrane reactors for natural gas conversion. Other applications include the partial oxidation of low-value hydrocarbons to high-value products, oxidative gas clean-up processes, and high-temperature oxygen separation.

18

Supported Flat Plate Thin Films for Oxygen Separation--Eltron Research, Inc., 4600 Airport Blvd., Boulder, CO 80301-3241; 303-530-0236

Dr. Richard Mackay, Principal Investigator Ms. Eileen E. Sammells, Business Official 
DOE Grant No. DE-FG03-99ER82783

Amount: \$749,922

Wide spread commercial production of fuels or chemicals from synthesis gas has been hindered by the high cost of producing oxygen, which is required for either the gasification of coal or the reforming of natural gas. Membrane processes for oxygen separation have shown potential, but polymeric membranes suffer from low selectivity, while ceramic membranes require high temperature to increase flux rate. In previous work, ceramic mixed ionic and electronic conductors were developed that exhibit high oxygen flux when placed in catalytic membrane reactors. These flux rates could be further increased by decreasing membrane thickness. Therefore, this project will develop an economical process for fabricating the ceramic membranes as thin films. Phase I developed a method for coating mixed polymer/powder precursors onto a porous substrate. The precursor decomposed and sintered to full density. The thin films were tested in reactors and demonstrated increased flux relative to thick membranes. Phase II will focus on the necessary processes for optimal reactor operation. Improved coating procedures for thin films will be developed and substrates with higher porosity will be fabricated. The performance of the catalyst will be optimized.

Commercial Applications and Other Benefits as described by the awardee: A quick, reliable, and inexpensive method of making ceramic thin films capable of separating oxygen from air should find use in applications requiring a source of pure oxygen. In combustion processes, for example, the use of pure oxygen will increase heating efficiency by the removal of the $\mathrm{N}_{2}$ diluent, with simultaneous reduction in $\mathrm{NO}_{\mathrm{x}}$ emissions.

\section{FUSION PLASMA SCIENCE RESEARCH}

19

\section{A Multi-Sensor Real-Time Resistive-Wall- Mode Identification Tool--FARTECH, Inc., 3146 Bunche Avenue, San Diego, CA 92122-2247; 858-455-6655 \\ Dr. Jin-Soo Kim, Principal Investigator \\ Dr. Jin-Soo Kim, Business Official \\ DOE Grant No. DE-FG03-99ER82791 \\ Amount: \$750,000}

The resistive-wall-mode instability limits the optimum operation of tokamak plasmas used in fusion energy research. The real-time identification and control of such deleterious modes are crucial for higher performance operation in present and future experiments, and therefore, for more economical fusion reactors. However, the identification and estimation of RWMs in a tokamak are difficult due to its slow, almost negligible, rotation speed and slow growth rate. This project will develop technology for utilizing signals from multiple diagnostics to identify the rotation, phase, and structure of resistive-wall-modes in real time. If the onset of the instability can be identified, the technology can be used in plasma feedback control. In Phase I, a computational tool was developed for resistive-wall-mode identification of moderotation, phase, and structure. In Phase II, the identification algorithm will be optimized for real-time analysis. The multi-sensor, real-time mode identifier will be conducted and its usefulness for RWM feedback control demonstrated at the DIII-D tokamak.

Commercial Applications and Other Benefits as described by the awardee: The multi-sensor computational tool that identifies the resistivewall-mode in real time should be an essential component of any system for controlling these instabilities and play a potentially large role in the success of the tokamak as a future fusion 
energy source. The technology could also be used to optimize the performance of any electromagnetic systems utilized in commercial industries, including electron beam or ion sources, plasma ashers, and other plasma processing devices.

\section{ENABLING TECHNOLOGIES FOR FUSION PLASMA EXPERIMENTS}

20

Power Supply for Plasma Heating--Diversified Technologies, Inc., 35 Wiggins Avenue, Bedford, MA 01730-2314; 781-275-9444

Dr. Marcel P. J. Gaudreau, Principal Investigator

Mr. Michael Kempkes, Business Official

DOE Grant No. DE-FG02-99ER82777

Amount: $\$ 749,699$

Future high power physics experiments will require an order of magnitude increase in power delivery over present systems. The cost and inefficiency of conventional power supplies is a significant issue in both the deployment and operation of future systems in the $10 \mathrm{MW}-200$ MW average power range. Improved power supply designs that are cost effective and efficient are critical to the technical and economic feasibility of fusion research. This project will leverage high voltage, solid state switching technology to design and build prototype high frequency $(5-20 \mathrm{kHz})$, high voltage, multimegawatt DC power supplies with significant improvements in life cycle cost over conventional $60 \mathrm{~Hz}$ power supplies. Phase I designed and built several $4 \mathrm{MW}$ buck regulator power supplies. A power control system was designed using switching buck regulators and solid state series protection switches (crowbar replacements). The reliability, efficiency, and cost of manufacturing were assessed for this system. In Phase II, a single string of the power control system, consisting of one buck regulator and two series klystron protection switches, will be built. The system will be installed at an operational fusion experiment, and its performance will be evaluated.
Commercial Applications and Other Benefits as described by the awardee: In a $200 \mathrm{MW}$ system, each percentage increase in power supply efficiency saves approximately $\$ 1 \mathrm{M} /$ year in electricity costs alone. This savings, and expected manufacturing cost reduction, should significantly reduce the cost of future high energy fusion systems and programs. Commercial applications of this technology include large ion implantation systems and pulsed electric field food sterilization.

21

A New Radiation Resistant Epoxy Resin System for Liquid Impregnation Fabrication of Composite Insulation--Eltron Research, Inc., 4600 Airport Boulevard, Boulder, CO 80301-3241; 303-530-0263

Dr. James B. Schutz, Principal Investigator

Ms. Eileen E. Sammells, Business Official

DOE Grant No. DE-FG03-99ER82782

Amount: $\$ 749,980$

Superconducting magnet coils are a major component of fusion plasma experiment devices. The electrical insulation in these magnet coils is typically an epoxy matrix composite, which must exhibit good mechanical strength and radiation resistance at cryogenic temperatures. The liquid epoxy must also have good processing characteristics for impregnating the magnet coils. Current epoxy systems do not combine good electrical and processing characteristics with mechanical strength and radiation resistance. Another major problem is the release of gases by the epoxy material during irradiation. This project will develop an epoxy resin formulation that exhibits good processing characteristics and reduced radiation outgassing. The formulation 
will be systematically optimized to further enhance processing properties, cryogenic mechanical strength, electrical characteristics, and radiation tolerance. In Phase I, epoxy resin, hardener and modifiers were selected based their radiation tolerance, processing characteristics, and mechanical performance. Phase II will fully characterize the properties of the optimized formulation, including critical cryogenic composite mechanical and electrical properties as well as the effects of radiation. The suitability of the formulation will be demonstrated by impregnation and evaluation of a large article that closely simulates the fabrication of a large magnet coil.

Commercial Applications and Other Benefits as described by the awardee: The epoxy resin formulation should find use as electrical insulation for cryogenic superconducting magnet systems, particularly those which will be subjected to irradiation. The formulation also should be of interest for cryogenic composite structural applications, as well as for commercial, industrial, and aerospace composites fabricated by liquid impregnation methods.

\section{2}

Advanced Heat Sink Materials for Fusion Energy Devices--Plasma Processes, Inc., 4914-D Moores Mill Road, Huntsville, AL 35811-1558; 256-851-7653

Mr. Timothy McKechnie, Principal Investigator

Mr. Timothy McKechnie, Business Official

DOE Grant No. DE-FG02-99ER82859

Amount: $\$ 750,000$

Cheaper and higher efficiency heat sinks are needed for future fusion energy devices. Existing heat sink materials do not allow for higher heat flux operation without costly efficiency enhancements for heat transfer in water- and gascooled components. This project will fabricate advanced heat sink materials, such as $\mathrm{CuCrNb}$, tungsten, molybdenum, and niobium alloys, to near net shape for high flux testing. Furthermore, heat transfer enhancements will be built into the internal cooling passages during heat sink formation. In Phase I, tungsten and molybdenum heat sinks were made for liquid-lithium-cooled components that are exposed to the plasma during the operation of a fusion system. High strength copper alloy heat sinks were made for the water-cooled components. Heat transfer enhancements (fins, flutes, etc.) were formed into the internal cooling passages. In Phase II, internal heat transfer enhancement features will be modeled, optimized and fabricated. Tungsten alloy heat sinks will be tested for liquid-metalcooled tokamaks, and high strength copper alloy heat sinks will be developed and tested for watercooled applications.

Commercial Applications and Other Benefits as described by the awardee: Applications for these heat sinks include heat pipes, sputtering targets for electronic manufacture, $\mathrm{x}$-ray targets for medical devices, industrial mold armor, high temperature protective coatings, rocket engines, valve surfacing, pump protection, combustors, chemical processing equipment, nose cones, power generation systems, and furnace components.

Fast Repetitive Arc Free Current Limiting Circuit Breaker--UTRON, Inc., 8506 Wellington Road, Suite 200, Manassas, VA 20109-3915; 703-369-5552

Dr. F. Douglas Witherspoon, Principal

Investigator

Mr. Dennis Massey, Business Official

DOE Grant No. DE-FG02-99ER82915

Amount: $\$ 749,768$

The Department of Energy (DOE) seeks fault protection devices that will effectively limit the current damage to radio frequency (RF) tubes during fault conditions associated with fusion plasma heating. This project will develop a new innovative circuit breaker capable of fast, arcfree current interruption. In a fault situation, the circuit breaker will limit the energy to RF tubes to less than a few joules at heating power levels of 10-50 MW. This was demonstrated experimentally in Phase $I$ on a small scale in a 
single shot mode by interrupting $11 \mathrm{kA}$ in less than 500 microseconds at about $13 \mathrm{MW}$. A current limiting circuit breaker device was fabricated utilizing a pulsed power source to separate the contacts with very high acceleration. This device successfully interrupted current in excess of $10 \mathrm{kA}$ at standoff voltages over $1 \mathrm{kV}$ in less than 500 microseconds. Experimentation with the Phase I device will be continued in the early part of Phase II. Then, a series of devices of ever increasing interruption performance will be built and tested. The goal of Phase II is to interrupt currents as high as $100 \mathrm{kA}$ at power levels as high as 10-50 MW in a few hundred microseconds.

Commercial Applications and Other Benefits as described by the awardee: Successful development of this device will result in faster arc-free opening switches/circuit breakers for both the commercial power industry and pulsed power applications, with longer component lifetimes, smaller physical size, and lower cost than currently available switches.

\section{ADVANCED TECHNOLOGIES AND MATERIALS FOR FUTURE FUSION SYSTEMS}

24

Hybrid Three-Dimensional SiC/C High Thermal Conductivity Composites--MER Corporation, 7960 South Kolb Road, Tucson, AZ 85706; 520-574-1980

Dr. Witold Kowbel, Principal Investigator

Dr. J. C. Withers, Business Official

DOE Grant No. DE-FG03-99ER82823

Amount: \$750,000

To provide improved strength and toughness, continuous fiber reinforced silicon carbide (SiC) composites (SiC-SiC), being developed primarily for advanced aerospace applications, are being examined for fusion reactor applications. Unfortunately, while the thermomechanical properties of SiC-SiC can be improved over that of monolithic $\mathrm{SiC}$, the thermal conductivity is significantly reduced. This project will develop a hybrid three-dimensional $\mathrm{SiC} / \mathrm{C}$ composite in which a high thermal conductivity carbon fiber ( $\mathrm{Z}$ direction) is used to improve the thermal conductivity. This system has the potential for improving through-the-thickness thermal conductivity, while maintaining composite properties upon irradiation. In Phase I, an SiC fabric, produced by chemical vapor reaction (CVR), was used for $X-Y$ reinforcement while $\mathrm{K}-1100$ fibers were used as $\mathrm{Z}$-reinforcement. A high conductivity $\mathrm{SiC}$-matrix, fabricated with a hybrid process that combines chemical vapor infiltration with polymer infiltration and pyrolysis, was used. A thermal conductivity of 65 meters/milli Kelvin was achieved through the thickness ( $\mathrm{Z}$ direction) of the matrix. In Phase II, process optimization for CVR-SiC and S-Nicelon fibers is planned, and irradiation resistance will be optimized.

Commercial Applications and Other Benefits as described by the awardee: The key benefit is for the fusion program. Commercial applications also exist in such energy related fields as heat exchangers. 


\section{ADVANCED CONCEPTS AND TECHNOLOGY FOR HIGH ENERGY ACCELERATORS}

25

\author{
Laser-Driven Cyclotron Autoresonance \\ Accelerator - LACARA--Omega-P, Inc., \\ 202008 Yale Station, Suite 100, New Haven, CT \\ 06520; 203-789-1164 \\ Dr. Jay L. Hirshfield, Principal Investigator \\ Mr. George P. Trahan, Business Official \\ DOE Grant No. DE-FG02-99ER82846 \\ Amount: $\$ 750,000$
}

High-energy electron acceleration using intense lasers, with gradients on the order of 100 $\mathrm{MeV} / \mathrm{m}$, for meter-length distances and in a manner that facilitates staging, has not yet been demonstrated. This project will develop laserdriven cyclotron autoresonance acceleration (LACARA) to exploit gyroresonance to achieve all of these characteristics. In Phase I, a thorough computational study was carried out to assess the effects of various parameters and design characteristics on beam acceleration. The computations showed that acceleration from 50 to $121 \mathrm{MeV}$ can occur over $150 \mathrm{~cm}$ in the focus of a two terawatt, 10.4 micron Gaussian laser beam with a minimum waist diameter of $0.20 \mathrm{~cm}$. An optimized design, employing an affordable single-coil dry cryomagnet, was developed for an experimental layout suitable for the Brookhaven National Laboratory Accelerator Test Facility (BNL-ATF). In Phase II, the final specifications for a single-coil, dry cryomagnet for a prototype LACARA will be determined and its procurement initiated. Design and fabrication will be undertaken for the LACARA beam pipe, including precision alignment fittings for copper mirrors and the pipe itself. Installation of the prototype LACARA in the BNL-ATF and evaluations of its performance will be carried out. Computations to enable the design of a future multi-stage LACARA will then be undertaken.
Commercial Applications and Other. Benefits as described by the awardee: A laser-based vacuum electron accelerator that can accelerate all particles in a short bunch equally, with a continuous acceleration gradient on the order of $100 \mathrm{MeV} / \mathrm{m}$ over meter-long paths and in a manner that facilitates staging, should be attractive to commercial uses of energetic electron beams. Applications include light sources and medical therapy.

Multi-Terawatt, 50fs Laser for Laser Accelerator Applications--Positive Light, Inc., 101 Cooper Court, Los Gatos, CA 95032-7604; 408-399-7744

Mr. Rimas Viselga, Principal Investigator

Mr. Jeremy Weston, Business Official

DOE Grant No. DE-FG03-99ER82860

Amount: $\$ 749,718$

The Department of Energy has a need for terawatt-class laser systems for advanced laserdriven particle acceleration applications.

Specifically, compact multi-terawatt systems are sought that are capable of providing at least 1 joule $(\mathrm{J})$ in 50 femtosecond (fs) single wavelength pulses or, alternately, $60 \mathrm{~J}, 4$ picosecond (ps) pulses in dual wavelength operation. This project will develop a compact terawatt chirped pulse amplifier laser system using titanium-doped sapphire (Ti:sapphire) as the gain medium to achieve short pulse widths. By combining a 10femtosecond seed laser and a cylindrical mirror pulse expander with two multi-pass amplifier stages, this system will generate multi-terawatt, 50 femtosecond pulses. Peak powers up to 5 terawatts at $10 \mathrm{Hertz}$ are feasible. If arrangements can be made to pump the final amplifier with a neodymium-doped glass laser developed earlier, then the goal of 50femtosecond pulses that exceed 20 terawatts will be achieved. In Phase I, the $\sim 110$ femtosecond, 
400 microjoule pulses from an available commercial ultrafast regenerative amplifier system were amplified to $\sim 155$ millijoules in a 16-mm diameter, four-pass Titanium sapphire amplifier. Based on these results, calculations show that further amplification would offer the potential for pulse energies up to 0.5 Joules at 10-Hertz. Phase II will develop and characterize a compact, chirped pulse amplifier system based on titanium-sapphire, tailored to laser-accelerator applications. This system will deliver peak powers greater than 5 terawatts at $10 \mathrm{Hertz}$. When combined with an existing neodymiumglass laser, peak powers greater than 20 terawatts should be possible. A secondary goal of Phase II will be to demonstrate the simultaneous amplification of two pulses at two closely spaced frequencies.

Commercial Applications and Other Benefits as described by the awardee: The availability of compact, relatively affordable laser-driven particle accelerators should lead to numerous potential commercial applications involving synchrotron optical sources, free electron lasers and sub-atomic particles. These applications include biomedical imaging, therapy, metallurgy and protein crystallography (accelerators) and surface science.

\section{7}

High-Performance

Three-Dimensional

Simulation Code for Laser Plasma Accelerators and Plasma Processing of Integrated Circuits, with a Graphical User Interface--Tech-X Corporation, 5541 Central Avenue, Suite 135, Boulder, CO 80301; 303-448-0727

Dr. David L. Bruhwiler, Principal Investigator

Dr. John R. Cary, Business Official

DOE Grant No. DE-FG03-99ER82903

Amount: $\$ 749,922$

Plasma wakefield accelerators can produce accelerating gradients many orders of magnitude higher than those of conventional structures, opening exciting possibilities for shorter and higher-energy electron linacs. However, in order 16 to maximize the potential of these accelerators, new particle simulation codes, incorporating new concepts in physics, are needed to support ongoing experiments and improve beam quality.

This project will develop a suite of 1-D, 2-D and 3-D particle-in-cell (PIC) simulation codes, with relativistic collision models. The 2-D and 3-D codes will simulate plasma accelerator experiments and new concepts, while the 1-D code will simulate radio frequency sheaths for industrial processing applications In Phase I, alternative approaches were evaluated, after which an existing PIC code was chosen. This code was run on the Cray T3E supercomputer, and a variety of new features were added, allowing for successful 2-D simulation of an ongoing plasma wakefield experiment. In Phase II, a 3-D version of the code will be further developed to make it suitable for laser-plasma accelerator simulations. Parallel input and output of large data sets, with sophisticated 3-D visualization of fields and particles, will be added to the 2-D and 3-D codes. These codes will be used to model plasma accelerator experiments and to study new accelerating concepts. A 1-D PIC code will be developed to simulate plasma sheaths in plasma processing experiments.

Commercial Applications and Other Benefits as described by the awardee: The codes developed may find utility in plasma processing and microwave electron tube industries. The 2-D and 3-D codes will be suitable for simulating a variety of plasma accelerator and microwave tube systems. 


\section{RADIO FREQUENCY ACCELERATOR TECHNOLOGY FOR HIGH ENERGY ACCELERATORS AND COLLIDERS}

28

\author{
Design of a $10 \mathrm{MW}, 91 \mathrm{GHz}$ Gyroklystron for \\ Linear Accelerators--Calabazas Creek \\ Research, 20937 Comer Drive, Saratoga, CA \\ 95070-3753; 408-741-8680 \\ Dr. Jeffrey Neilson, Principal Investigator \\ Dr. R. Lawrence Ives, Business Official \\ DOE Grant No. DE-FG03-99ER82754 \\ Amount: $\$ 750,000$
}

In recent design scenarios for advanced linear colliders with center-of-mass energies above 1 $\mathrm{TeV}$, the expected radio frequency (RF) drive frequency is typically above X-Band. Unfortunately, in terms of peak power, the capability of current RF source technology is several orders of magnitude below anticipated requirements. It has been well demonstrated in the past that gyrotrons significantly outperform conventional tubes in the millimeter regime. This project will utilize gyroklystron technology with state-of-the-art X-Band klystrons to develop an RF source at $91.392 \mathrm{GHz}$ that has a peak power capability about 3 orders of magnitude above the state-of-the-art for linear tubes. In Phase I, a design was developed that provides $10 \mathrm{MW}$ of RF power while maintaining all parameters (voltage gradients, power loadings, mode purity, emission density, and field strengths) within acceptable limits. In Phase II, the design will be completed, and a prototype gyroklystron will be built and tested. Phase II will result in a source that will be capable of testing W-Band accelerator components and (with pulse compression) capable of driving a W-Band accelerator structure.

Commercial Applications and Other Benefits as described by the awardee: High power W-Band sources should have applicability at research centers to test the viability of W-Band accelerator components in terms of breakdown fields, peak and average power capabilities, etc.
The tube could also power an accelerator structure to look at issues such as dark current. Other potential applications include land- and ship-based radars, medical accelerators, and materials processing.

Low Field Solenoid Periodic Focusing of Accelerator Klystrons--California Tube Laboratory, Inc., 125 Aviation Way, Watsonville, CA 95076-2058; 831-761-1199

Mr. David E. Blank, Principal Investigator Mr. Peter S. Campbell, Business Official DOE Grant No. DE-FG03-99ER82755 Amount: $\$ 748,190$

A large reduction in the number of klystrons and modulators ( $4: 1$, even $6: 1)$ is required to reduce the cost of the Next Generation Linear Collider (NLC). In turn, this will require an equivalent increase in the average power per klystron. Therefore, multiple beam klystrons (MBKs) must be used. This project addresses the solution to the major problem with MBKs, off-axis focusing. In principle, solenoid periodic magnet (SPM) focusing will solve the off-axis focusing problem, while greatly reducing the solenoid power required to focus the beams. Phase I designed a SPM beamstick that is compatible with the SLAC XL4 klystron geometry. The beamstick was fabricated and preliminary tests were carried out, both on and off-axis, in three configurations -- one with the solenoid alone and two with SPM stacks of different periodic length. Whole onaxis focusing was comparable for the three configurations, the SPM configurations required far less solenoid power. Off-axis focusing was far superior with the SPM stacks. In Phase II, two test vehicles will be designed, fabricated and tested in collaboration with SLAC. The first will be a beam tester to establish the entrance conditions and SPM stack parameters for $99 \%$ beam transmission. The second will be one 
module of the MBK, with $12.5 \mathrm{MW}$ peak RF output, $50 \mathrm{~dB}$ gain, $250 \mathrm{kV}$ beam voltage and 62 percent efficiency.

Commercial Applications and Other Benefits as described by the awardee: The use of SPM focusing for reduced solenoid power and MBK off-axis focusing should be applicable to any accelerator as well as to high power radars. The savings to the NLC could be as much as $\$ 100 \mathrm{M}$.

\section{0}

New Concepts for Pulsed Power Modulators-Diversified Technologies, Inc., 35 Wiggins Avenue, Bedford, MA 01730-2314; 781-275-9444

Dr. Jeffrey Casey, Principal Investigator Mr. Michael A. Kempkes, Business Official DOE Grant No. DE-FG02-99ER82775

Amount: $\$ 700,000$

Future particle accelerators and colliders will require orders of magnitude improvement in pulsed power generation and control. These systems are projected to require millions of volts and thousands of amperes for very short pulses. Current pulsed power technology utilizes large gas discharge tubes, pulse forming networks, and pulse transformers. However, these technologies are incapable of scaling to the peak power requirements of the next generation of high power physics systems. In this project, previously developed solid state pulse power systems, up to $140 \mathrm{kV}$ and $2000 \mathrm{~A}$, will be scaled-up by nearly an order of magnitude in voltage. A prototype modulator with very high voltage and very high power will be built to demonstrate and assess the scalability of this technology. Phase I has developed two new designs for very high voltage solid state switching. The approaches for scaling have been proven on a small scale. The effort was then focussed on the development and characterization of a $500 \mathrm{kV}$ solid state switch, which does not require pulse transformers. The designs have been assessed for cost, efficiency, and reliability. Phase II will build and characterize the modulator design for $500 \mathrm{kV}$ Next Generation Linear Collider (NLC) operation. This will be followed by a complete segment of the $500 \mathrm{kV}$ modulator being built and characterized under representative operative conditions.

Commercial Applications and Other Benefits as described by the awardee: In addition to accelerators, these pulsed power systems should be applicable to new materials modification and treatment processes, and sterilization of both food and medical equipment. Very high voltage systems, may enable the development of new manufacturing and materials processing techniques across a wide range of industrial and medical applications.

\section{HIGH-FIELD SUPERCONDUCTOR AND SUPERCONDUCTING MAGNET TECHNOLOGIES FOR HIGH ENERGY PARTICLE COLLIDERS}

\section{1}

\author{
Co-Processed Ceramic Insulation for High \\ Field Accelerator Magnets--Composite \\ Technology Development, Inc., 1505 Coal Creek \\ Drive, Lafayette, CO 80026-2782: \\ 303-664-0394 \\ Mr. John A. Rice, Principal Investigator \\ Dr. Naseem A. Munshi, Business Official
}

DOE Grant No. DE-FG03-99ER82766

Amount: $\$ 600,000$

Some high performance superconducting magnets, such as wind-before-react systems, cannot be manufactured because of the temperature limits of current organic-based insulation materials. This limitation forces the magnet designer to accept either lower performance or higher manufacturing costs. A 
ceramic-based insulation that is stable at the processing temperatures would eliminate this problem. This project will develop a ceramic composite insulator that offers the ease of application and processing of conventional organic insulation but is able to withstand the same heat treatment as the superconducting wire itself. In Phase I, two new ceramic materials were identified which have the potential to meet existing performance requirements at a substantially lower cost. No adverse chemical incompatibilities were observed between the wire and insulation, even at very high temperatures and over long periods of time. The thermal expansion and strain behavior of conductor/ceramic insulation composites proved to be better than with traditional epoxy insulation. Phase II will optimize the ceramic matrix and fabric to reduce fabrication costs. The mechanical, adhesion, thermal, and electric properties of the insulation will be measured, and its performance in conductor/ceramic insulation composite coil stacks will be characterized.

Commercial Applications and Other Benefits as described by the awardee: The high temperature stability of the ceramic insulation would eliminate complex coil fabrication steps, lowering production costs. Applications such as high field magnets, fusion magnets, and medical MRI instruments would become more viable with improved magnet processing, higher magnetic field strength, and greater reliability.

32

Low-Cost, Long-Length Manufacturing of Continuously Melt-Quenched Processed, Full Stabilized, Multifilamentary High-Field Nb3Al Superconductor-Plastronic, Inc., Subsidiary of Eurus Technologies Inc., 2031. E. Paul Dirac Drive, Innovation Park, Tallahassee, FL 32310; 1-800-574-1800

Mr. Michael Tomsic, Principal Investigator

Mr. John A. Romans, Business Official

DOE Grant No. DE-FG02-99ER82789

Amount: $\$ 600,000$
The economical manufacture of long lengths of multifilament niobium-aluminum $\left(\mathrm{Nb}_{3} \mathrm{Al}\right)$ wire is needed to produce very high magnetic field coils for nuclear magnetic resonance, accelerators, and research magnets. Such a wire is required for future high-energy accelerator magnets because $\mathrm{Nb}_{3} \mathrm{Al}$ maintains a high critical current density in fields in the 10-25 Tesla range. This project will develop a low cost manufacturing process for $\mathrm{Nb}_{3} \mathrm{Al}$ superconducting wire in lengths of 300 meters or more. In Phase I, long continuous tubes were successfully filled with a mechanically-alloyed powder, a unique wire drawing technique produced $\mathrm{Nb}_{3} \mathrm{Al}$ precursor wires, and a rapid quench process was applied to the wire. Following the rapid quench, a process and associated equipment were developed for adding a copper stablizer before the ordering heat treatment. The Phase II effort will further develop the continuous tube forming and filling process as well as the process for reducing the precursor wire. Ultimately, the production of $300+$ meter lengths of monofilament and multifilament $\mathrm{Nb}_{3} \mathrm{Al}$ wire will be demonstrated.

Commercial Applications and Other Benefits as described by the awardee: The technology should provide low cost, higher performing superconducting wire for High Energy Physics applications like accelerators. Commercial applications include NMR, MRI, Magnetic Separation, and R\&D Magnets.

\section{3}

Improvement of High Field Performance and Reliability of $\mathrm{Nb}_{3} \mathrm{Sn}$ Conductor by Powder-InTube (PIT) Method--Supercon, Inc., 830 Boston Turnpike, Shrewsbury, MA 01545-3301; 508-842-0174

Mr. Terrence Wong, Principal Investigator

Mrs. Elaine Tarkiainen, Business Official

DOE Grant No. DE-FG02-99ER82896

Amount: $\$ 600,000$

In order for the next generation high energy physics particle accelerators to reach higher collision energy levels, there is a need for higher field dipole magnets. This in turn demands 
superconductors that have higher critical current densities in the presence of high magnetic fields, than can be typically reached with the best materials available today. $\mathrm{Nb}_{3} \mathrm{Sn}$ conductors are the most promising material for the next generation of dipole magnets with fields $>15$ Tesla; however further improvements are necessary. This project will develop a high critical-current-density $\mathrm{Nb}_{3} \mathrm{Sn}^{-}$conductor produced by the powder-in-tube (PIT) method, with tantalum and titanium additions used to improve the upper critical field and high field critical current density. Phase I produced $\mathrm{NbSn}_{2}$ powder for use in the PIT process. Tantalum- and titanium-doped niobium tubes were produced by wrapping thin layers of sheets around a removable core and using hot extrusion to bond the components together. Finally, billet assembly and drawing techniques were established to produce multifilamentary composites. Phase II will further improve the PIT process by improving $\mathrm{NbSn}_{2}$ powder homogeneity and reducing maximum particle size. The powders will be used to produce multifilamentary composites with final filament diameters between 40-100 microns. Finally, heat treatment studies will be conducted to improve the critical current density of the Ta- and Ti-doped samples.

Commercial Applications and Other Benefits as described by the awardee: Higher performing $\mathrm{Nb}_{3} \mathrm{Sn}$ conductors would be used in high field dipole magnets for high energy physics. Other uses include magnetic confinement systems for fusion reactors and nuclear magnetic resonance (NMR) systems for chemical analysis.

\section{4}

High Performance Nb3Sn (Ta) by Tin Enrichment and Increased Filament Content-Superconducting Systems, Inc., 90 Rumford Avenue, Waltham, MA 02453; 781-642-6702

Dr. Shahin Pourrahimi, Principal Investigator

Dr. Shahin Pourrahimi, Business Official DOE Grant No. DE-FG02-99ER82898

Amount: $\$ 600,000$
The construction of next generation particle accelerators for high energy physics require that $\mathrm{Nb}_{3} \mathrm{Sn}$ superconductors exhibit unprecedented performance and a substantial cost reduction. In order to meet these needs, this project will develop an innovative route to the fabrication of high-tin ( $\mathrm{Sn}$ ) content, bronze matrix wire with niobium-tantalum $(\mathrm{Nb}(\mathrm{Ta})$ ) filaments. The high Sn content will allow for increased filament content within the wire and lead to $\mathrm{Sn}$-rich $\mathrm{Nb}_{3} \mathrm{Sn}$ (Ta) filaments with high performance. During Phase I, a novel and economical approach to $\mathrm{Nb}_{3}$ $\mathrm{Sn}$ wire fabrication was demonstrated by fabricating high performance $\mathrm{Nb}_{3} \mathrm{Sn}(\mathrm{Ta})$ wires produced by tin enrichment with increased filament content. A non-copper critical current density of $2100 \mathrm{~A} / \mathrm{mm} 2$ at $15 \mathrm{~T}$ and $4.2 \mathrm{~K}$ was achieved. In Phase II, the wire fabrication technology will be scaled up, wires for both highcritical-current density and low $\mathrm{AC}$ losses will be designed, and high current $\mathrm{Nb}_{3} \mathrm{Sn}$ cables will be fabricated.

Commercial Applications and Other Benefits as described by the awardee: The $\mathrm{Nb}_{3} \mathrm{Sn}$ (Ta) superconductors should find application in next generation particle accelerators, energy storage, fusion reactors, and in NMR (nuclear magnetic resonance) magnets.

A High Current Density Very Low Cost Niobium Tin (Titanium) Doped Conductor Utilizing A Novel Internal Tin Process, with Separate Stabilizing Elements Scalable to Modern Niobium Titanium Production Economics--Supergenics, 1233 Tree Bay Lane, Sarasota, FL 34242; 941-349-0930

Mr. Bruce A. Zeitlin, Principal Investigator

Mr. Bruce A. Zeitlin, Business Official

DOE Grant No. DE-FG02-99ER82899.

Amount: $\$ 600,000$

Magnets for next generation accelerators require higher performance from the superconductors used in their construction, so that magnetic fields of 12 Telsa or more can be achieved. The superconductors must also be cost effective in order 
for the magnets generating these fields to be economically viable. This project will develop a $\mathrm{Nb}_{3} \mathrm{Sn}$, superconductor using an internal tin process approach. The approach will utilize micron-size filaments doped with titanium to achieve high current in a process that will allow the use of large-scale production techniques, such as those utilized in NbTi. The resultant conductor will be a cable of low cost copper, combined with the superconductor, that can readily be assembled into required cable configurations. Phase I demonstrated that long lengths of high-current-density wire could be fabricated using the large scale production techniques. Current densities in the superconducting layer reached almost 5600 $\mathrm{A} / \mathrm{mm} 2$ at $12 \mathrm{~T}$ in a non-optimized conductor Cost models were developed that estimated production costs would be approximately
$\$ 1.25 / \mathrm{KA}$-meter in large quantities. In Phase II, the conductor will be designed and fabricated to optimize the ratios of tin and niobium for improved current density. Enough of the conductor will be produced to validate the process and permit its evaluation in magnets. A cladding technique will be also be developed. Finally, a production-scale billet will be designed and fabricated into wire to evaluate production parameters.

Commercial Applications and Other Benefits as described by the awardee: Lower cost superconductors should reduce the cost and expand the range of MRI, a billion dollar industry. In addition, applications in the utility sector that have been considering HTS superconductors may find that low cost $\mathrm{Nb}_{3} \mathrm{Sn}$ may be suitable in the nearer term.

\section{TECHNOLOGIES FOR THE NEXT-GENERATION ELECTRON-POSITRON LINEAR COLLIDER}

36

Automated Diamond Turning Lathe for the
Production of Copper Accelerator Cells--DAC
Vision, Inc., 6390 Rose Lane, Carpinteria, CA
93013; 805-684-8307
Mr. James Bryan, Principal Investigator
Mr. James W. Drain, Business Official
DOE Grant No. DE-FG03-99ER82774
Amount: $\$ 750,000$

Copper accelerator cells, like many sub-micron tolerance metal parts, are now manually produced on research quality, single-point, diamond-turning lathes. The process is slow and costly, and finished part quality is inconsistent due to required human intervention. In this project, a high volume production, diamondturning lathe for sub-micron tolerence metal parts will be built. It will feature automated operation and will be optimized for the mass production of copper accelerator cells. In Phase $I$, the specifications and conceptual design of the machine was completed. Problems concerning thermal stability, geometric error reduction, part holding accuracy, repeatability at the sub-micron level, automated part loading and unloading, and rapid cutting speeds were addressed. In Phase II, the machine will be built and tested. Performance will be demonstrated by cutting and evaluating actual parts.

Commercial Applications and Other Benefits as described by the awardee: The automated turning lathe could reduce the cost of machining accelerator cells for the next generation linear collider by an order of magnitude, potentially saving tens of millions of dollars. This tool should also be valuable to other industries that require precision metal parts, such as those used in disk and tape drives, precision optics, and medical implants. 
High Power Switch--Diversified Technologies, Inc., 35 Wiggins Avenue, Bedford, MA 01730-2314; 781-275-9444

Dr. Jeffrey Casey, Principal Investigator Mr. Micheal A. Kempkes, Business Official DOE Grant No. DE-FG02-99ER82778 Amount: \$627,596

High power switching in high energy physics applications currently uses vacuum and gas tube technologies, such as thyratrons and ignitrons. However, the Next Generation Linear Collider will require the use of new solid-state switch technology, which has the potential to satisfy aggressive performance goals with lower cost and improved reliability. This project will utilize proven insulated gate biopolar transistor switching technology to replace the tubes in Pulse Forming Network (PFN) pulsing and crowbar protection systems with fast, solid state, series switches. In Phase I, high power switches, capable of replacing thyratrons in accelerator applications and scalable to the needs of other colliders and high power applications, were designed. The control systems required to provide fast response to arc conditions were prototyped. In Phase II, a complete $100 \mathrm{kV}, 50 \mathrm{~A}$ $\mathrm{CW}$ opening switch system will be prototyped and tested in collaboration with Argonne National Laboratory (ANL). This switch will be evaluated as a possible replacement for ANL's mercury ignitron crowbar system at the laboratory's Advanced Photon Source. Methods for reducing the unit cost of a solid state opening switch will also be addressed.

Commercial Applications and Other Benefits as described by the awardee: Many of the drawbacks of existing thyratron and ignitron circuits -- cost, lack of reliability, and use of hazardous materials -- could be eliminated by using solid state switches. High power, solid state switches could save millions of dollars in capital and operating costs in future accelerators and in commercial operations, such as plasmabased materials modification and semiconductor fabrication.

22
Hybrid NLC Modulator--Diversified Technologies, Inc., 35 Wiggins Avenue, Bedford, MA $01730-2314 ; 781-275-9444$

Dr. Jeffrey Casey, Principal Investigator

Mr. Michael A. Kempkes, Business Official

DOE Grant No. DE-FG02-99ER82776

Amount: \$749,691

The Next Generation Linear Collider (NLC) will require 1600 klystron modulators capable of providing $500 \mathrm{kV}, 530$ A pulses at $120 \mathrm{~Hz}$, representing one of the largest NLC cost factors. Modulators with the lowest possible life cycle cost are needed for this program to proceed in a cost-effective, realizable manner. This project will utilize high voltage, solid state technology to design and build hybrid modulators (solid state switch/pulse transformer) with significantly improved efficiency, reliability, and life cycle compared to conventional modulators. In Phase I, the hybrid NLC modulator was designed, and the key technological capabilities necessary to build it were demonstrated. The cost, reliability, and efficiency were assessed and compared to other NLC modulator architectures. This design was optimized for low life cycle cost. Phase II will build a full specification prototype for the hybrid modulator, incorporating low cost manufacturing techniques and advanced state switch and transformer designs for high efficiency. After construction and evaluation, the prototype will be provided to the Stanford Linear Accelerator Center for assessment.

Commercial Applications and Other Benefits as described by the awardee: The hybrid modulator should be significantly less expensive to build and operate than conventional modulators and should have nearly an order of magnitude higher reliability. Reliable, low cost pulsed power should be applicable to many commercial processes, including semiconductor fabrication, metal surface treatments, medical diagnostic and treatment systems, and food sterilization. 
Adiabatic Forming of Copper Accelerator Cells for the Next Generation Linear Collider-LMC, Inc., 333 Dietz Avenue, De Kalb, IL 60115; 815-758-3514

Mr. Lennart J. Lindell, Principal Investigator

Mr. Lennart J. Lindell, Business Official

DOE Grant No. DE-FG02-99ER82813

Amount: $\$ 100,000$

The linear accelerator for the next generation electron-positron linear collider (NLC) will be comprised of nine thousand accelerator structures, each requiring 207 ultra-high precision copper cells. The cost of these nearly two million copper cells is expected to be as much as ten percent of the total cost of the entire machine. In order to reduce the cost of manufacturing these cells, this project will apply adiabatic forming technology to eliminate the rough machining of parts from conventional milling and turning machines. The adiabatic process can mold exotic lightweight metals into shapes not possible with conventional presses, requires little or no lubricants or cutting fluids, and reduces or eliminates cleaning and waste disposal. In Phase I, calculations required to adiabatically form cells were made and a production press was developed, leading prototype parts based upon an actual scaled cell. Metallography and chemistry analyses were performed to document crystal size and uniformity, as well levels and types of chemical residuals introduced during the forming process. In Phase II, a full size press, based upon the operating principles of the smaller press developed and used in Phase I, will be designed, built, tested, and used to produce prototype parts.

Commercial Applications and Other Benefits as described by the awardee: The adiabatic forming process could be the technology of choice for producing the accelerator cells for the NLC. It is extremely fast (milliseconds), reduces or eliminates many manufacturing steps, and uses 80 percent less energy than conventional methods.

\section{HIGH ENERGY PHYSICS DETECTORS}

40

Low Cost Support Structures, with New Advanced Composite Materials Tailored for Ultra-Stable Particle Tracking Detectors-HYTEC, Inc., 110 Eastgate Dr., Suite 100, Los Alamos, NM 87544-3304; 505-662-0080

Mr. William O. Miller, Principal Investigator

Mr. William O. Miller, Business Official

DOE Grant No. DE-FG03-99ER82801

Amount: \$674,063

Precision charged-particle detectors for high energy physics research rely upon ultra-highmodulus composite materials. These high performance materials satisfy the need for stability and low mass, and possess a high radiation length that limits deleterious interactions with the charged-particles. However, as detector sizes increase, the high costs of the composites materials themselves and the equally expensive graphite-fiber honeycomb core materials become prohibitive. This project will develop new material processing techniques for producing high stiffness sandwich panels from inexpensive composite materials. A very lowdensity, high modulus syntactic carbon foam material will also be developed as a possible replacement to the graphite-fiber honeycomb core. In Phase I, new material processing techniques were developed to allow ultra-high modulus carbonized laminates to be produced from low cost materials. A sandwich panel was constructed that significantly reduced the mass of the pixel detector panel without a significant impact on stiffness. An investigation of the processing steps needed to produce carbonized syntactic foam was initiated. Phase II will 
complete the material testing for the low-cost thin carbon-carbon laminates in order to qualify the material as an acceptable replacement to the resin-based composites for particle tracking detectors used in the next generation linear collider (NLC). Also, low cost, high stiffness-toweight ratio sandwich panels will be developed using the syntactic carbon foam material.
Commercial Applications and Other Benefits as described by the awardee: The new material processing techniques should produce particle detector sandwich facings with 60 percent greater stiffness and overall cost savings of 50 percent. Applications include stable structures for next generation physics detectors, passive cooling of high heat flux electronics, and lightweight spacebased optical structures requiring passive cooling.

\section{NUCLEAR PHYSICS INSTRUMENTATION AND TECHNIQUES}

41

SQUID Susceptometers for Read Out of Magnetic Microcalorimeters--Hypres, Inc., 175 Clearbrook Road, Elmsford, NY 10523-1109; 914-592-1190

Dr. Masoud Radparvar, Principal Investigator

Dr. Elie Track, Business Official

DOE Grant No. DE-FG02-99ER82800

Amount: $\$ 749,974$

A magnetic calorimeter is an attractive candidate for an x-ray detector for nuclear physics research. This project will develop an array of magnetic microcalorimeters based upon the use of magnetic sensors to measure very small temperature changes resulting from the absorption of $x$-rays in a telescope. The magnetization of localized paramagnetic ions in a metallic matrix will be determined by using a DC Superconducting Quantum Interference Device (SQUID), which is extremely sensitive and ideally adapted to the measurement of flux changes. The resultant sensitivity of this calorimeter will be only weakly dependent upon heat capacity and should exhibit a very fast response. In Phase I, a new fabrication process was developed, and ultra-low SQUID susceptometers were designed, fabricated, and integrated with magnetic microcalorimeters for $\mathrm{x}$ ray measurements. In Phase II, an array of magnetic microcalorimeters will be developed and integrated with an array of these SQUID susceptometers. The goal is to demonstrate a complete imaging system with resolution of $2 \mathrm{ev}$ at $6 \mathrm{keV}$ for the focal plane of an $\mathrm{x}$-ray telescope.

Commercial Applications and Other Benefits as described by the awardee: The availability of a magnetic microcalorimeter, integrated with a low-cost SQUID susceptometer, should have many nuclear and high energy physics research applications. In addition, the SQUID readout can be used for biomagnetometry for the mapping of brain functions, and the detection of corrosion and cracks in aircraft.

\section{2}

\section{Nuclear Data Evaluation Tools--Scientific Digital Visions, Inc., 2 North Second Street, Suite 1215, San Jose, CA 95113-1303; 408-289-8494 \\ Ms. Tracy Langlands, Principal Investigator \\ Ms. Tracy Langlands, Business Official \\ DOE Grant No. DE-FG03-99ER82880 \\ Amount: $\$ 748,977$}

The U.S. and International nuclear data networks have identified a critical need for new technologies for nuclear data evaluators. Out-ofdate methods are currently used within the networks, and this has been identified as a possible barrier for attracting young scientists. This project will develop advanced methods for data entry, define an extensible markup language 
(XML) data transmission format for nuclear data, and develop a model for a server-based data checking and physics checking code. Also an interactive-level scheme will be developed to both display nuclear data in an electronic document and allow evaluators to extract that data. Phase I produced prototypes for data entry and level scheme software. Data parsing tools were developed to aid in the study of data formats. A model was developed for an advanced data checking system, and tests were performed on the feasibility of converting data checking and physics checking code into the Java programming language. The intent of Phase II is to produce comprehensive data entry and level scheme software. A suite of data checking and physics checking codes will be converted to Java and a server-based data checking system for Web services will be constructed.

Commercial Applications and Other Benefits as described by the awardee: In addition to their application to the National Nuclear Data Center, the data parsing and XML technologies have direct applications in Web services, providing important methods and formats for data transfer. Data entry and data checking technologies will support advanced Web and application user interfaces.

\section{NUCLEAR PHYSICS ACCELERATOR TECHNOLOGY}

43

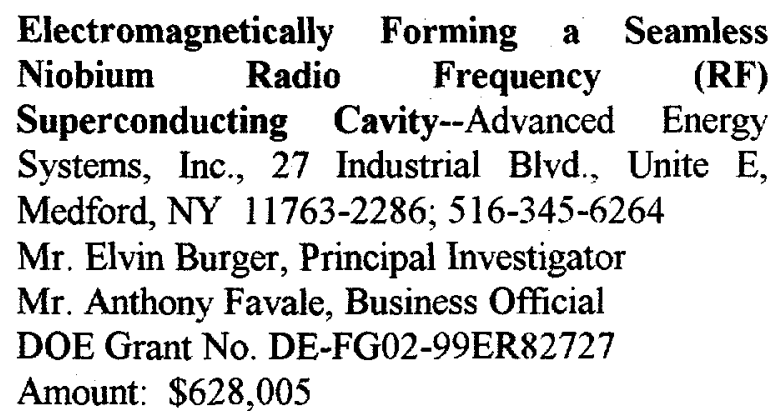

This project will further develop the process of electromagnetically forming a niobium radio frequency (RF) superconducting cavity for nuclear physics accelerators. This process eliminates costly machining and electron beam welding and improves performance characteristics. Phase I used a finite element analysis to determine the power required in the magnetic coils that would be used for forming the cavity, as well as the arrangement of the coils with respect to the workpiece and mandrel. An axisymmetric transient magnetic model was developed and used to determine eddy currents, power loss due to these eddy currents, and induced magnetic forces in the niobium workpiece, all as a function of time. These results were used to develop a detailed design of the tooling required to electromagnetically form a complete superconducting RF cavity. The Phase II project will fabricate a seamless niobium RF superconducting cavity and test it at the Department of Energy's Jefferson Laboratory Cryogenic Facility.

Commercial Applications and Other Benefits as described by the awardee: Niobium exhibits superconductivity, that is, the lack of electrical resistance at very low temperatures. As a result, niobium forming should be useful in electron beam accelerators, power generation, propulsion, and other electronic devices. 
the accelerator world by allowing much higher power input per unit length than currently

Development of High Power RF Windows for Next-Generation Superconducting and Normal Conducting Accelerators--Advanced Energy Systems, Inc., 27 Industrial Blvd., Unite E, Medford, NY 11763-2286; 631-345-6264

Mr. Michael Cole, Principal Investigator

Mr. Anthony Favale, Business Official DOE Grant No. DE-FG02-99ER82725

Amount: $\$ 635,411$

High current, high duty factor accelerators have historically been plagued by the problem of transferring the necessary radio frequency (RF) power through the vacuum boundary between the RF transmission system and the high vacuum environment of the accelerator. The lower the maximum power transmitted per window, the more the windows required to deliver a certain amount of power. Therefore, a higher power window assembly means that fewer windows would be needed to deliver a certain amount of power; this would reduce the length (and hence cost) and complexity of the accelerator while improving its reliability. This project will integrate electromagnetic, thermal, and structural analysis with novel mechanical design, material utilization, and manufacturing techniques to develop a window assembly that combines high thermal conductivity, effective ceramic cooling, optimized ceramic shape, and optimized waveguide details. The goal is a window that is not limited by thermal effects. In Phase I, proofof-process specimens were designed and fabricated to prove that all the necessary steps in the fabrication process could be achieved. In parallel, a detailed analysis of operational requirements for two candidate windows was executed, leading to a detailed design of a window built for the Department of Energy's Jefferson Laboratory accelerator. Phase II will design, fabricate, and test two statistically significant sets of windows for two window configurations.

Commercial Applications and Other Benefits as described by the awardee: A window assembly not limited by thermal effects would revolutionize 26 possible. For superconducting systems in particular, significantly higher gradients with higher current could be produced, thereby improving overall cost, efficiency, and reliability of accelerator systems.

45

Superconducting RF Photocathode Electron Gun--Advanced Energy Systems, Inc., 27 Industrial Blvd,, Unite E, Medford, NY 11763-2286; 631-345-6264

Mr. Micheal Cole, Principal Investigator

Mr. Anthony J. Favale, Business Official

DOE Grant No. DE-FG02-99ER82724

Amount: $\$ 740,765$

High-brightness, superconducting, continuous wave accelerators presently use an electron source that is separated from the initial accelerating modules. Significant gains in compactness, beam brightness, efficiency, and reliability could be realized by developing a superconducting gun where the photocathode is an integral part of the initial accelerating cavity. This project will develop a system that eliminates the need for a separate electron gun by utilizing the cavity endwall itself as the integral photocathode. Using existing cryogenic, laser, radio frequency, and diagnostic equipment, the achievement of superior brightness and beam quality from the gun will be demonstrated. Phase I designed and fabricated a representative test specimen and measured the quantum efficiency of the candidate photocathode and demonstrated that a significant electron beam power can be emitted. Simulations showed that a high quality electron beam can be generated using this photocathode and that the resulting beam can be accelerated through a superconducting structure. A superconducting cavity system was designed for testing in Phase II. Phase II will continue the photocathode development and quantum efficiency testing, as well as the design of an integrated superconducting assembly, and test it at the Department of Energy's Brookhaven National Laboratory. 
Commercial Applications and Other Benefits as described by the awardee: A superconducting photocathode gun would be an ideal source for superconducting electron accelerators and free electron lasers. Additional applications would ensue if the gun can be further developed towards the higher power required for commercial material processing and military free-electron-laser applications due to the improved cost, performance, and efficiency.

\section{6}

High Power RF Window and its Input Coupler Technology--AMAC International, Inc., Applied Research Center, 12050 Jefferson Avenue, Suite 348, Newport News, VA 23606-4323; 757-249-3595

Dr. Quan-Sheng Shu, Principal Investigator

Ms. Loren Loving, Business Official

DOE Grant No. DE-FG02-99ER82739

Amount: $\$ 750,000$

The RF input window and associated couplers in superconducting cavities must operate through various power levels and RF loads of complex impedances without arcing, overheating, or nonuniform mechanical stress failures. This project will develop RF coupler and window technology that can operate at high power with significant improvements in reliability and cost. In Phase I, two prototype windows were constructed using an innovative compression ring and an internal cooling jacket. A laser technique was developed to weld stainless steel to copper for the final vacuum seal. Calculations of the interference fit indicated that the window was under $15,000 \mathrm{psi}$ compression at room temperatures. In Phase II, the innovative compression ring, internal cooling, and laser welding/brazing will be optimized and implemented to both waveguide windows and coaxial windows, and new window processing technologies (glow discharging cleaning and high temperature baking) will be investigated to improve post-processing quality and efficiency. The window-coupler system will be further developed to reduce post-processing time and cost. The window-coupler technology will be tested at high power and operating conditions.
Commercial Applications and Other Benefits as described by the awardee: RF windows using the compression ring, internal cooling jacket technology and laser welding/brazing should carry higher RF power and have enhanced reliability compared to other current $\mathrm{RF}$ window designs. The innovations should have applicability in every RF input coupler of all the accelerator projects world-wide, such as SNS, JLab upgrade, Muon Collider, and TESLA. The technology could also be used in gyrotron windows and other vacuum joints of dissimilar materials.

\section{7}

\section{A High Efficiency Electron Cyclotron Resonance Ion Source System for the Production of Radioactive Ion Beams-- Berkeley Ion Equipment, Inc., 3400 De La Cruz Boulevard, Suite V, Santa Clara, CA 95054-2609; 408-727-9071 \\ Dr. Zu Q. Xie, Principal Investigator \\ Mr. M. Wei, Business Official \\ DOE Grant No. DE-FG03-99ER82750 \\ Amount: \$750,000}

This project will fabricate and characterize a high efficiency, high charge state, permanent magnet electron cyclotron resonance ion source. This ion source system will be used for the production of rare isotope ion beams and other heavy ion accelerators for nuclear science research supported by the Department of Energy. Phase I determined a set of optimum parameters for the ion source and developed a hot liner and a medium temperature oven for the production of solid isotopes. The engineering design and the cost estimates for the ion source system were completed. In the design, the ion source was placed at an adequate distance from the production target, through a thermal transfer line, to reduce radiation damage to the ion source magnets. Phase II will fabricate the permanent magnet electron cyclotron resonance ion source system designed in Phase I to demonstrate and characterize its high ionization efficiency and other capabilities. Tests will be conducted to determine the ability of the ion sources to 
produce intense intermediate and high charge state heavy ion beams in continuous and pulsed modes. The tests will be conducted at the Department of Energy's Argonne National Laboratory to demonstrate the high ion efficiencies of the ion source.

Commercial Applications and Other Benefits as described by the awardee: In addition to the applications in nuclear and atomic physics and applied science, the electron-cyclotron-resonance ion source should find application in industry for high energy ion implantation, ion milling, and surface modification.

Development of New Lossy Material for Cryogenic and Ambient Applications-Ceradyne, Inc., 3169 Red Hill Avenue, Costa Mesa, CA 92626; 714-549-0421

Dr. Biljana Mikijelj, Principal Investigator

Mr. Howard George, Business Official

DOE Grant No. DE-FG03-99ER82759

Amount: \$456,057

Current, commercially available microwave absorbing materials do not provide adequate microwave absorption at the extremely low temperatures (approaching absolute zero) that are required for operation in many super-cooled, superconductor-based particle accelerators. Specifically, the new Next Linear Collider requires a high volume of a lower cost lossy material for incorporation into its accelerator cells. This project will develop a commercial source of lossy materials, based on a ceramic aluminum-nitride (AIN) matrix, with temperature-independent properties. In Phase I, two cryogenically lossy material combinations were identified and confirmed to have temperature-independent dielectric loss properties under cryogenic conditions. Both materials are vacuum compatible and have been bonded to copper. Phase II will initiate material optimization and manufacturing scale-up for these materials. Both material densification hotpressing and near-net-shape pressureless sintering techniques will be evaluated. Relevant 28 material properties will be measured, and process capability will be determined.

Commercial Applications and Other Benefits as described by the awardee: The material should have lossy properties at extremely low temperatures for applications such as CEBAF. Larger markets for these materials include microwave tubes both for military applications and for high energy particle accelerators.

\section{9}

A High Force Actuator for SRF Cavity Tuning--Energen, Inc., 17 D Sterling Road, Billerica, MA 01862-2518; 978-671-5400

Dr. Chad H. Joshi, Principal Investigator

Dr. Chad H. Joshi, Business Official

DOE Grant No. DE-FG02-99ER82784

Amount: $\$ 750,000$

Superconducting radio frequency (SRF) cavities, used in high- and medium-energy physics research, are tuned by mechanically squeezing them along their axis. The tuner actuator must produce a force of several thousand pounds and have submicron positioning resolution. This project will develop an SRF tuning mechanism based on newly developed cryogenic magnetostrictive materials; these materials have saturation strains of $0.5 \%$ and can be used to build compact actuators. In Phase I, a high force linear stepper motor employing magnetostrictive materials was designed and built. It had a motion range of 10 millimeters, an output force capability of $2200 \mathrm{~N}$ (500 lbs), and submicron positioning resolution. The force capability was limited by the static frictional brake of the clamps of the stepper motor. A mechanical leverage system was designed to amplify the force capability to provide the $22 \mathrm{kN}(5000 \mathrm{lbs})$ force necessary to tune the SRF cavities for the Department of Energy Jefferson Laboratory upgrade. Phase II will develop more cost-effective techniques for manufacturing the magnetostrictive materials, and their mechanical, magnetic, electrical and thermal properties will be characterized. The goal is to design and build stepper motor tuners capable of providing a wide tuning range. 
Commercial Applications and Other Benefits as described by the awardee: These magnetostrictive tuners should provide improved performance of superconducting RF cavities used in particle accelerators, free electron lasers and synchrotron radiation light sources. Other applications include adaptive optics, materials processing, and medicine.

\section{WASTE CHARACTERIZATION}

50

Next-Generation, Portable XRF System-Photon Imaging, Inc., 19355 Business Center Drive, Suite 8, Northridge, CA 91324-3503; 818-709-2468

Dr. Jan S. Iwanczyk, Principal Investigator

Dr. Bradley E. Patt, Business Official

DOE Grant No. DE-FG03-99ER82853

Amount: $\$ 750,000$

A truly portable $x$-ray fluorescence (XRF) analyzer would significantly help reduce the cost of treatment and disposal of hazardous metal contaminants by providing improved characterization technology. This project will develop an XRF analyzer with unprecedented accuracy. By using an increased sampling frequency, statistical errors will be reduced. Not only will there be significant reductions in cost and time, environmental and personnel risks will be reduced by eliminating manual sample taking, transportation, and laboratory analysis of contaminated material. Phase I designed and constructed a next-generation, miniature x-ray spectrometer that incorporated a novel largearea, silicon drift $\mathrm{x}$-ray detector and a thermoelectric cooler. $\mathrm{X}$-ray response to wide range of energies (1.5-25 keV) showed comparable energy resolution to that of cryogenically-cooled germanium or lithiumdoped silicon detector systems. In Phase II, the finalized XRF instrument will be developed. It will contain an optimized detector, excitation sources, processing electronics, and supporting software for automated data collection and XRF analysis.

Commercial Applications and Other Benefits as described by the awardee: New x-ray detectors and XRF instruments with high energy resolution, high count rate throughput, and near room temperature operation could open up numerous important market opportunities in environmental contamination monitoring, process control and materials identification, and electron column instruments.

\section{1}

An Advanced Avalanche-Photodiode Based Spectroscopic Radiation Monitor--Radiation Monitoring Devices, Inc., 44 Hunt Street, Watertown, MA 02472-4699; 617-926-1167

Dr. Mitchell Woodring, Principal Investigator

Dr. Gerald Entine, Business Official DOE Grant No. DE-FG02-99ER82866

Amount: $\$ 750,000$

The Department of Energy must reduce the time for treating and disposing of radioactive wastes from facilities undergoing decontamination and decommissioning. Currently, site assessment and monitoring is too slow and too costly because the required analysis techniques are located off-site. This project will design, develop, and fabricate a high sensitivity radiation imager that is directly applicable to the decontamination and decommissioning of its facilities. The device will be based upon the development of a novel detector, a dual-diode-backed silicon avalanche photodiode (APD). This detector was designed, fabricated, and tested in Phase I. The ability to acquire energy spectra with the $2 \times 2$ diode-backed APD array coupled to a sodium-doped cesium scintillator was demonstrated while operating in a row-column addressing arrangement. Collimated gamma-ray sources were used to demonstrate the position sensitivity of the device. A signal processing, storage, and control system was 
developed and used to examine the performance of the array. In Phase II, the radiation imaging system will be further refined, and system field tests will be conducted to determine performance and operational characteristics.

Commercial Applications and Other Benefits as described by the awardee: An instrument with the ability to image radioactive sources could find use at nuclear research facilities, hospitals and clinics, nuclear power generation plants, nuclear fuel production facilities, and similar locations where radioactive sources are used or can be found.

\section{WASTE TREATMENT AND STABILIZATION}

\section{2}

Separation of Metal Ions from Liquid Waste Streams--Applied Sciences, Inc., 141 West Xenia Avenue, P.O. Box 579, Cedarville, OH 45314-0579; 937-766-2020

Dr. D. Gerald Glasgow, Principal Investigator Mr. Max L. Lake, Business Official DOE Grant No. DE-FG02-99ER82744 Amount: \$674,064

Metal ions, such as uranium, strontium, cadmium, and transuranics, pose a threat to the environment because of their toxicity and radioactivity. This project will develop a new electrosorption technique based on carbon nanofiber electrodes for removing metal ions from waste streams. Competing methods are not useful because the electrolyte typically dissociates before substantial electrosorption of metals such as uranium can occur. Phase I demonstrated a reduction of uranium concentration from 100 parts per million to less than 20 parts per billion using special nanofiber electrodes. A capacity of over 10 grams of uranium per gram of cathode was achieved. The electrodes were reversible and reusable, and the energy cost was as low as $900 \mathrm{~J} /$ gram of uranium absorbed. Phase II will focus on design, manufacturing, testing, and validating a full-scale unit capable of remediating wastes at contaminated Department of Energy sites.

Commercial Applications and Other Benefits as described by the awardee: Applications include remediation of uranium-containing streams at DOE and DOD facilities, as well as at uranium mines, including decommissioned mines. Spinoffs should also occur for direct groundwater remediation, including the removal of other metals.

\section{3}

Enhanced Selective Leaching of Chromium from Radioactive Sludges--Lynntech, Inc., 7610 Eastmark Drive, Suite 202, College Station, TX 77840-4023; 979-693-0017

Dr. Jinseong Kim, Principal Investigator

Mr. Oliver J. Murphy, Business Official DOE Grant No. DE-FG03-99ER82817

Amount: $\$ 683,605$

Techniques are needed for the safe and costeffective remediation of radioactive wastes stored in more than 300 underground storage tanks at Department of Energy sites. The presence of chromium materials in the waste sludge is a major problem because it has a relatively large impact on the volume of high level waste (HLW) when vitrified. The cost of vitrification and storage could be substantially reduced by effectively separating chromium from the HLW using a selective oxidant combined with an enhanced selective oxidation process to reduce the amount of chromium in these wastes. This project will develop a selective oxidative alkaline leaching (ESOAL) process to accomplish this removal. In Phase I, the process was tested with simulant waste and shown to be capable of 
removing chromium species from complex sludges with a 100 percent efficiency in a short operation time. Efficiencies and removal rates were substantially better than other processes such as caustic leaching and conventional oxidative leaching. The ESOAL process was also successful in the co-dissolution of nonradioactive species (with a corresponding high reduction of sludge mass) with no dissolution of strontium, an important radioactive species found in underground storage tanks. In Phase II, the process will be optimized and tested with several sludge simulants as well as with actual waste from the Hanford site. A scaled-up automated process will then be developed and tested.

Commercial Applications and Other Benefits as described by the awardee: Besides its application to the remediation of nuclear waste, this process could be applied to the treatment of many wastewaters, such as from the dye, electroplating, and pharmaceutical industries as well as municipal sewage.

\section{TECHNOLOGY FOR TREATMENT, MANAGEMENT, AND DISPOSITION OF URANIUM HEXAFLUORIDE}

54

\author{
Production of High Value Fluorine Gases for \\ the Semiconductor Industry--Starmet Cor- \\ poration, 2229 Main Street, Concord, MA \\ 01742; 978-369-5410 \\ Dr. John Bulko, Principal Investigator \\ Mr. Donald T. King, Business Official \\ DOE Grant No. DE-FG02-99ER82894 \\ Amount: $\$ 739,025$
}

DOE is responsible for managing nearly 1.5 billion pounds of uranium hexafluoride stored in carbon steel cylinders in Ohio, Kentucky, and Tennessee. The cylinders are corroding and beginning to become an environmental concern. The cost of converting these cylinders to a more stable form, such as an oxide, is estimated at about $\$ 2$ billion. If valuable chemicals can be produced from the fluorine portion, the overall management cost to DOE and the taxpayers will be reduced. This project will develop technology to extract fluorine from uranium hexafluoride and produce high value, high purity gases for use in the semiconductor industry. These gases (tungsten hexafluoride and germanium hexafluoride) are used for chemical vapor deposition in semiconductor manufacturing. Phase I demonstrated that germanium and tungsten oxides would react with uranium tetrafluoride to produce the respective gaseous metal fluorides. In addition to studying the basic reactions, the sensitivity of the conversion process to temperature, heating rate, and in some cases, variations in the starting material were also investigated. Phase II will develop a production process for generation, capture, and purification of $\mathrm{GeF}_{4}$ to the point that it is market-ready for existing semiconductor applications. Phase II will also include engineering development and scaleup of processes for $\mathrm{WF}_{6}$ manufacture.

Commercial Applications and Other Benefits as described by the awardee: Successful implementation of this project will produce titanium metal, a high-value metal product that can be sold as a powder or ingot, and a fluoride product (cryolite or HF). Both products should be used in very large quantities by industry. 


\section{ADVANCED ENVIRONMENT MONITORING TECHNOLOGY}

55

Fiber Optic Grating Based Moisture Sensor-Blue Road Research, 2555 N. E.205th Avenue, Fairview, OR 97024-8600; 503-667-7772

Mr. Whtten Schultz, Principal Investigator

Mr. Eric Udd, Business Official

DOE Grant No. DE-FG03-99ER82753

Amount: $\$ 750,000$

It is difficult, but necessary, to monitor the movement of water through a waste containment area. The movement of the water could act as a carrier to contaminate groundwater, or could act as a source of corrosion. This project will develop a low cost (less than $\$ 100$ per sensor in 1,000 part lots), distributed fiber grating moisture sensor capable of monitoring 50 points on a single fiber line or 500 points with a $1 \times 10$ switch. Phase I developed and demonstrated a moisture sensor that was small (dimensions within $8 \times 8 \times 20 \mathrm{~mm}$ ), reversible, and capable of continuous moisture measurement. Sensitivity levels showed promise of meeting the 1 percent volumetric target. The Phase II project will develop improvements in absolute accuracy and stability of the sensors while reducing overall costs. Interfaces will be developed suitable for supporting the deployment of the sensors at a landfill, bridge, or aerospace platform, and an actual system will be deployed at both a landfill and bridge.

Commercial Applications and Other Benefits as described by the awardee: The moisture system for monitoring hazardous waste areas should be applicable for use on civil structures such as bridges and to the aerospace community.

\section{ATMOSPHERIC MEASUREMENT AND SAMPLING TECHNOLOGY}

56

Development of III-Nitride UV Detectors-Avyd Devices, Inc., 2925 College Avenue, Unit A-1, Costa Mesa, CA 92626; 714-751-8553

Dr. Honnavalli R. Vydyanath, Principal Investigator

Dr. Honnavalli R. Vydyanath, Business Official DOE Grant No. DE-FG03-99ER82748

Amount: \$749,961

High sensitivity, ultraviolet (UV) detectors for radiometric instrumentation are needed to monitor atmospheric chemical composition changes. This project will develop technology for 111-nitride based UV detectors with extremely high sensitivity and very efficient rejection of the longer wavelength radiation. In Phase I, molecular beam epitaxy (MBE) was used to grow p-i-n structures on sapphire substrates upon which $\mathrm{GaN}$ buffer layers had been grown using metal oxide chemical vapor deposition (MOCVD). The $\mathrm{p}-\mathrm{i}-\mathrm{n}$ devices were tested and a more efficient rejection of longer wavelength radiation was demonstrated in these structures compared to others with no MOCVD-grown GaN buffer layers. Phase II will optimize the approach and demonstrate multiplexed p-i-n detector arrays $(256 \times 256)$ with high reproducibility. The detectors will have response times on the order of a nanosecond or less, detectivity greater than $10^{12}$ watt $^{-1}$, or noiseequivalent-power less than 1 picowatt $(\mathrm{pW})$ for $1 \mathrm{~Hz}$ bandwidth and UV/visible rejection ratio of $10^{6}$ or higher at room temperature.

Commercial Applications and Other Benefits as described by the awardee: Applications include missile plume detection for military use, environmental monitoring, automobile engine 
combustion sensing, remote sensing of earth resources, solar astronomy and burner monitoring in gas turbines.

\section{7}

Mercury Cadmium Telluride (HgCdTe) Detectors for Near Infrared Applications-Avyd Devices, Inc., 2925 College Avenue, Unit A-1, Costa Mesa, CA 92626; 714-751-8553

Dr. Honnavalli R. Vydyanath, Principal Investigator

Dr. Honnavalli R. Vydyanath, Business Official DOE Grant No. DE-FG03-99ER82747

Amount: $\$ 600,000$

Short wavelength infrared detectors, operating at near theoretical limits for radiometric instrumentation, are needed to monitor changes in atmospheric chemical composition. This project will develop $\mathrm{HgCdTe}$ photovoltaic devices that are sensitive in the short wave region of solar radiation and exhibit detection performance close to the theoretical limits imposed by the material itself. In Phase I, HgCdTe photodiode arrays were demonstrated with cut-off wavelength of 2 microns, $R_{0} A$ performance of $3 \times 10^{3} \cdot \mathrm{ohm}-\mathrm{cm}^{2}$, and external quantum efficiency of $69 \%$ at $300 \mathrm{~K}$. At zero bias, these performance values translate to a detectivity in excess of $5 \times 10^{11}$ Jones at room temperature. Phase II will optimize and reproducibly demonstrate 256 x 256 mosaics hybridized to Silicon Read Out Integration chips.

Commercial Applications and Other Benefits as described by the awardee: Applications include detection of hot boxes on railroad cars, process control, measurement of the temperature of brake linings, power lines, cutting tools, welding and soldering operations, detection and monitoring of air pollution, and skin thermography for tumor detection and infrared cell sorting, etc.
Low Temperature, High Altitude Humidity Sensor--Nanomaterials Research Corporation, 2620 Trade Center Avenue, Longmont, CO 80503-7551; 303-702-1672

Dr. Peter Mardilovich, Principal Investigator

Dr. Tapesh Yadav, Business Official

DOE Grant No. DE-FG03-99ER82839

Amount: $\$ 750,000$

Significant improvements in the measurement of atmospheric parameters are required for longterm atmospheric monitoring to support sustainable and pollution-free energy developments of the future. In particular, a new approach to humidity sensing from balloon launched radiosondes is required to provide high accuracy and time resolution for measurements at very high altitudes and temperatures down to $60^{\circ} \mathrm{C}$. This project will develop novel microsensors and sensor arrays for long term, low temperature, and high altitude humidity monitoring. The approach is based on a novel, low-cost, nanoporous material with tunable chemical composition and nanoengineered porous morphology. Phase I demonstrated that nanofabricated, high sensitivity, low power, lowcost, humidity sensors can provide superior performance than currently available sensors down to $\sim 60^{\circ} \mathrm{C}$. Phase II will further develop, test, and optimize the technology for the high performance humidity monitoring microsensors in high altitude conditions. Manufacturing technology will be further developed and commercialization possibilities will be pursued.

Commercial Applications and Other Benefits as described by the awardee: The primary application of nano-engineered sensors is in low power atmospheric monitoring of humidity. Spinoff applications include reliable humidity sensors for cryogenic applications, process engineering, and consumer applications. 
LC scanners and holographic filters can be critical components of helmet-mounted displays, fax machines, and equipment for spectroscopy and nuclear facilities.

A Multi-Holographic Filter with a Liquid Crystal Scanner for Surface Optical Reflectance--Physical Optics Corporation, 20600 Gramercy Place, Building 100, Torrance, CA 90501-1821; 310-320-3088

Mr. Kevin Yu, Principal Investigator

Mr. Gordon Drew, Business Official

DOE Grant No. DE-FG03-99ER82855

Amount: $\$ 600,000$

Measurement of solar radiation over a wide spectral range is necessary to effectively monitor the earth's atmosphere. Current radiometers capable of measuring light intensity in multiple wavelength bands are bulky, heavy (when using moving parts such as mechanical scanners or rotating arms) and, because of optical filter drift, cannot produce consistent results, especially for long term operation under field conditions. This project will develop a compact, low cost, lightweight Surface Optical Reflectance Meter (SORM) based on a thin stack of environmentally stable reflection holographic filters and a lightweight two-dimensional liquid crystal panel. The system will address such problems as drift, sensitivity, field-of-view, wide spectral range, and compatibility with existing system components. The Phase I project designed the SORM components, analyzed their performance, verified the concept by experimental demonstration, and investigated the fabrication process by which the processed system can be manufactured. Feasibility of the proposed concept was demonstrated. Phase II will optimize the design and develop a fullyoperational SORM prototype system, including the scanner and the stacked holographic filters.

Commercial Applications and Other Benefits as described by the awardee: The lightweight, compact and low cost SORM system should benefit a variety of practical applications in remote sensing, environmental monitoring, climate research, and pyrometry for manufacturing process control, target recognition, and product inspection. In addition, 34
60

\section{A Low-Cost Automated Radiosonde Launch System--Yankee Environmental Systems, 55833-99-II Inc., 101 Industrial Boulevard, Turners Falls, MA 01376-1608; 413-863-0200 Mr. William M. Stein, Principal Investigator Ms. Cynthia A. Cote, Business Official DOE Grant No. DE-FG02-99ER82920 Amount: $\$ 750,000$}

Recent concern over widespread global warning has highlighted the need for improving upper atmospheric measurements of temperature, humidity, and winds. These measurements are required daily at hundreds of locations around the world and are primarily made by radiosondes that are carried aloft by balloons and tracked by ground stations. Currently, radiosonde setup and launch is performed manually by trained operators. Also, no mechanism exists to autocalibrate the radiosondes just prior to launch, resulting in insufficiently precise data. Although one commercially available automated launch system does exist, its $\$ 350,000$ price makes it impractical for most programs. This project will develop a low cost automated radiosonde launch system that is robust and fault-tolerant. Once loaded, for each cycle it will automatically: (I) power up a radiosonde; (2) check the radio frequency link; (3) start data acquisition; (4) perform a calibration of the radiosonde temperature and humidity signals; (5) inflate a balloon and verify inflation; (6) release the balloon; (7) verify that the payload is successfully away; (8) continuously capture data for duration of flight; (9) transfer data to a central data archive; and (10) return to an idle state and prepare for the next launch. In Phase I, a preliminary system was prototyped and tested outdoors, validating specifications for the launcher and key components. The Phase II project will complete a detailed engineering system design for a field-ready automated launch 
system. Three levels of engineering prototypes will be developed and tested with particular emphasis on system reliability, especially under high wind conditions. The goal is to be able to automatically precalibrate and launch anywhere from 8 to 24 radiosondes without human intervention.

Commercial Applications and Other Benefits as described by the awardee: The technology should be applicable to weather forecasting and climate monitoring programs, as well for climate change research. The worldwide market for such systems is roughly $\$ 30-50$ million. It should provide a significant improvements in radiosonde launch capability for programs such as DOE's Atmospheric Radiation Measurement program, various NASA programs, and the National Weather Service.

\section{CARBON CYCLE MEASUREMENTS OF THE ATMOSPHERE AND THE BIOSPHERE}

61

\author{
A Diode Laser Sensor for High Precision \\ Measurement of Terrestrial $\mathrm{CO}_{2}$ Sources and \\ Sinks--Physical Sciences, Inc., 20 New England \\ Business Center, Andover, MA 01810-1077; \\ 978-689-0003 \\ Dr. David M. Sonnenfroh, Principal Investigator \\ Dr. Byron David Green, Business Official \\ DOE Grant No. DE-FG02-99ER82858 \\ Amount: \$749,758
}

High precision measurements of the sequestration and emission of $\mathrm{CO}_{2}$ and other greenhouse gases from various ecosystems, as well as from anthropogenic sources, are required for improved climate and climate change predictions. Existing instrumentation is insufficient to the need in sensitivity, temporal response, chemical selectivity, or ability to be routinely used in the field for large area survey measurements. This project will develop and test a new, room temperature diode-laser-based sensor for eddy covariance measurements of $\mathrm{CO}_{2}$ and $\mathrm{H}_{2} \mathrm{O}$ simultaneously and will be easily field deployable for extended periods. In addition, its unique air probe can be replicated to form networks of sensors, controlled by a single central processor, for gradient or large area surveys. The Phase I project designed and fabricated an in situ, open path, air sampling probe and combined it with an existing control system. A precision of 0.4 percent over 70 minutes while monitoring 333 ppmv $\mathrm{CO}_{2}$ was demonstrated. Phase II will fabricate the field prototype laser sensor based on the design produced during Phase I, perform field tests, and then compare it with existing nondispersive infrared (NDIR) sensors.

Commercial Applications and Other Benefits as described by the awardee: The sensor should find application in atmospheric field research concerned with quantifying the increasing burden of greenhouse gases in the atmosphere and their impact on global climate change. The underlying high speed, high precision, multiple-trace gas measurement technology should also have commercial application in industrial process control, agricultural monitoring, fence line fugitive emissions monitoring, compliance monitoring, and combustion analysis. 


\section{MEDICAL APPLICATIONS}

62

\author{
Rapid Processing of Positioning Information \\ for Hand-Held Gamma Camera--PEM \\ Technologies, Inc, 5611 Roosevelt Street, \\ Bethesda, MD 20817-6739; 301-564-0835 \\ Dr. Irving Weinberg, Principal Investigator \\ Dr. Irving Weinberg, Business Official \\ DOE Grant No. DE-FG02-99ER82852 \\ Amount: \$598,433
}

Medical and industrial users of noninvasive radiation detection technology want improved contrast and depth information to better visualize subtle abnormalities. This project will improve the detection and characterization of radioactive sources employed in surgery. The improvement can be achieved cost-effectively through novel sensor technologies and appropriate algorithms. In Phase I, a working human-directed imaging device was constructed. Enhanced contrast of the radioactive sources was demonstrated, compared to conventional cameras. The Phase II project will develop algorithms to improve realtime performance, modify the hardware for intraoperative use, conduct clinical trials to validate the technology, and examine novel applications for radioactive waste detection.

Commercial Applications and Other Benefits as described by the awardee: The product is aimed at the robust and growing intra-operative visualization market. A potential Phase III partner has been identified for both medical and industrial applications.

\section{3}

Small Animal SPECT Camera for Quantitative Molecular Medicine--Photon Imaging, Inc., 19355 Business Center Drive, Suite 8, Northridge, CA 91324-3503; 818-709-2468

Dr. Lawrence MacDonald, Principal Investigator
Dr. Bradley E. Patt, Business Official

DOE Grant No. DE-FG03-99ER82854

Amount: $\$ 750,000$

In vivo animal studies are extremely valuable in the development of new diagnostic and therapeutic pharmaceuticals. Unfortunately adequate instrumentation does not exist to accurately image the distribution of these pharmaceutical agents in small animals. Through the development of solid-state gamma-ray detectors, new instrumentation could provide image and energy resolution that far surpasses existing technology. This project will implement solid-state design into a high resolution, threedimensional rodent-imaging gamma camera for this application. The Phase I project developed sophisticated computer modeling of the physics of the high resolution system and performed experimental measurements on individual components and miniature prototypes. The results showed that the proposed technology was feasible. In Phase II, a full prototype rodent imaging tomographic gamma will be constructed by coupling a large-area detector array to high density readout electronics and software for system control and image processing. Rodent imaging using newly developed pharmaceuticals will be conducted with the prototype.

Commercial Applications and Other Benefits as described by the awardee: The product will meet a growing commercial demand for systerns capable of facilitating the development of pharmaceuticals for the study and treatment of neurological disease, oncology, cardiology, and other applications. The system should have improved performance, compactness, and lower cost compared to existing technology. 


\section{GENOME, STRUCTURAL BIOLOGY, AND RELATED BIOTECHNOLOGIES}

64

\author{
A Visual Data-Flow Editor Capable of \\ Integrating Data Analysis and Database \\ Querying--CyberConnect Corporation, 131 \\ Hunting Lodge Road, Storrs, CT 06268-1331; \\ 860-429-2666 \\ Mr. Ravi Nori, Principal Investigator \\ Mr. Karl Beckert, Business Official \\ DOE Grant No. DE-FG02-99ER82773 \\ Amount: \$749,995
}

In order to determine mapping sequence variations or polymorphism between homologous genomic regions, a genome scientist must be able to access genomic data from different sources and use many data analysis and visualization programs. To achieve this, software must be developed to automate tedious and repetitive data handling, database querying, and analysis tasks.

This project will develop a data-flow editing environment in which genome scientists with minimal computer training can easily describe data analysis tasks. The software tool will allow the scientist to organize and coordinate individual data retrieval tasks from different data sources, and combine them with data analysis to derive answers to biologically significant questions. Phase 1 demonstrated the feasibility of a dataflow editing environment in which interactions between data access and data analysis can be freely described by genome scientists with minimal computer training. Phase II will convert the Phase I prototype into a robust, full-fledged system deployable in a distributed environment, and establish the feasibility of operating an Internet-based service with the proposed visual data-flow analysis tool. The tool will be developed as independent software that can be readily linked to existing analysis and visualization programs with little or no prior modifications.
Commercial Applications and Other Benefits as described by the awardee: This software should be immediately usable by the molecular biology and pharmaceutical industries, both of which are becoming more computationally intensive. Since data-flow management problems are not unique to computational biology, the software should also be applicable to other data and computationally intensive areas, e.g., physics, chemistry, engineering, and finance.

\section{5}

\author{
Genome Analysis Tools and Information \\ Services--Genome Informatics Corporation, \\ 1020 Commerce Park Drive, Oak Ridge, TN \\ 37830; 423-220-0043 \\ Mr. Doug Hyatt, Principal Investigator \\ Dr. Ed Uberbacher, Business Official \\ DOE Grant No. DE-FG02-99ER82794 \\ Amount: $\$ 600,000$
}

Knowledge about genomes is rapidly becoming a central driver for product development in pharmaceutical and biotechnology industries. Many companies lack the tools, infrastructure and information to take full advantage of the flood of genome data and would greatly benefit from easy-to-access genome analysis tools and information about genome content. This project will develop and deploy a suite of analysis tools and a series of information services that will provide valuable insights into genes and gene function. The Phase I project restructured a gene discovery code for higher modularity, improved algorithms, and computational efficiency. In addition, a Java interface was constructed, databases were packaged for support of the software, and a command-line applications programming interface was created. Phase II will develop a comprehensive genome analysis tool suite and pipeline for deployment at remote sites, a data warehousing system that allows users to manage and integrate these data at their sites, and 
software to provide users with views of annotated genomes. These tools and services will be deployed via public and private secure networks.

Commercial Applications and Other Benefits as described by the awardee: These software tools should allow the sequence data produced by DOE and other federal agencies to be used for important medical and environmental applications such as diagnostics, drug design, and bioremediation. The use of these tools should also further the development of the rapidly growing U.S. industry segment focused on biotechnology, bioengineering, and genetic medicine.

66

Versatile Liquid Crystal Tunable Interference Filter for Chromosome Analysis--Physical Optics Corporation, 20600 Gramercy Place, Building 100, Torrance, CA 90501-1821; 310-320-3088

Dr. Tin Aye, Principal Investigator

Mr. Gordon Drew, Business Official

DOE Grant No. DE-FG03-99ER82856

Amount: $\$ 749,998$

High resolution spectral imaging microscopy using multi-probe fluorescence in situ hybridization (FISH) has been demonstrated as a potential technique for chromosome analysis. However, current spectral imaging systems are limited in the number of chromosomes that can be probed simultaneously. This project will develop a highly versatile, high resolution, wide spectral range, high speed, liquid crystal tunable interference filter (LCTIF) to overcome the limitations of current spectral imaging techniques used with FISH. The Phase I project designed the liquid crystal tunable interference filter components, analyzed their performance, and verified the concept by experimental demonstrations. Also, the fabrication process by which the proposed device can be manufactured was investigated, and the feasibility of the concept was demonstrated. Phase II will optimize the design and develop a fullyoperational prototype system for the liquid crystal tunable interference filter with high speed tunability over a broad spectral range (from visible to near infrared), with high out-of-band rejection. The system will be tested and evaluated in terms of its suitability for future DOE multiprobe gene mapping projects and for commercial applications.

Commercial Applications and Other Benefits as described by the awardee: Commercial applications include tunable spectral filters for spectral imaging microscopy, and other multispectral imaging sensor applications, both for sale to OEMs and as stand-alone instruments. Its low cost, high speed, and broadband continuous tuning make it suitable for spectral sensing in field use, including in hazardous environments and factories.

\section{METAL FORMING}

67

\author{
A Generic Approach to Improved Semi-Solid \\ Forming of Metals--Chesapeake Composites \\ Corporation, 239 Old Churchman's Road, New \\ Castle, DE 19720-1529; 302-324-9110 \\ Mr. Eric M. Klier, Principal Investigator \\ Dr. Alexander Brown, Business Official \\ DOE Grant No. DE-FG02-99ER82763 \\ Amount: $\$ 748,890$
}

The full potential of the semi-solid forming process (with its requirement for a uniform, spherical primary phase throughout) has not been realized due to difficulties in developing technology for the production of large inexpensive feedstock. Furthermore, even with existing semi-solid metal systems, narrow process windows and alloy chemistry restrictions increase process costs and limit performance attributes. This project will develop production 
technology for semi-solid forming utilizing new proprietary feedstock metals. The approach offers an intrinsic, highly uniform, spherical solid phase, without electromagnetic or mechanical shearing. The Phase I project successfully demonstrated that large diameter feedstock with a uniform permanent spherical solid phase, could be produced at low cost. Also, the semi-solid forming process window was expanded and semisolid material was formed with substantially enhanced physical and mechanical properties compared to existing semi-solid forming materials. The Phase II project will produce 6inch diameter billets suitable for semi-solid forming (SSF) and design, fabricate, and test aluminum engine components. Primary billet processing costs, SSF costs, heat and mass transport during SSF, and material behavior will be modeled. Both basic and application-specific microstructural/mechanical/thermal property databases will be developed. Finally, 4-inch billet SSF capability will be established

Commercial Applications and Other Benefits as described by the awardee: The semi-solid forming process should apply to high volume, cost sensitive components and components with higher performance requirements. The total volume of semi-solid formed components should increase in such applications as automotive thermal management and brake components, military engine components, electronic packaging, and precision equipment.

High-Strain-Rate Superplastic Forging of Aluminum Alloys--Materials Modification, Inc., 2929 P-1 Eskridge Road, Fairfax, VA 22031-2213; 703-560-1371

Dr. R. Radhakrishnan, Principal Investigator Dr. T.S. Sudarshan, Business OfficialDOE Grant No. DE-FG02-99ER82825

Amount: $\$ 750,000$

Currently, superplastic forging is limited to low strain rates $\left(10^{-3}-10^{-3} \mathrm{~s}^{-1}\right)$ and to the forming of aerospace components. It is limited by the high cost associated with thermomechanical and recrystallization processes, and the long time ( $\geq 30$ minutes) required to form near-net shapes.

This project will demonstrate an economical synthesis and processing technique for $\mathrm{Al}-\mathrm{Cr}-\mathrm{Fe}$ alloys and superplastically form them to near-net shape. The superplastic behavior of these alloys will be studied and used to design and fabricate near-net shape parts in a short cycle time $(<10$ min). The Phase I project synthesized nanocrystalline aluminum, chromium, and iron nanopowders. These nanopowders were alloyed using high-energy ball milling and superplastically forged in less than 10 minutes. In Phase II, nanocrystalline Al-Cr-Fe alloys will be synthesized directly. Fundamental understanding of the effect of powder particle characteristics (particle size, size distribution and morphology) on the consolidation and superplastic forming of these alloys will be evaluated. These data will be used to fabricate fuselage fairings, pulley sprockets and rocker arms.

Commercial Applications and Other Benefits as described by the awardee: High-strain-rate superplastic forging is expected to result in economically-viable, rapid near-net-shape forming technique for the automobile, aerospace, and semiconductor industries. 


\section{PROCESSING FOR SURFACE HARDNESS}

69

Functionally Graded, Nanocrystalline, Multiphase, Boron- and Carbon-Based Superhard Coatings--Spire Corporation, One Patriots Park, Bedford, MA 01730-2396; 781-275-6000

Dr. Fereydoon Namavar, Principal Investigator

Dr. Richard S. Gregorio, Business Official

DOE Grant No. DE-FG02-99ER82891

Amount: \$749,921

Industrial and medical components require harder surfaces to increase durability and life. Attempts to coat metallic components with diamond or ceramic films, such as for earth drilling equipment, have failed due to the poor adherence of dissimilar materials and the brittleness of the films: This project will fabricate functionally graded, nanocrystalline, multiphase, superhard, adherent, low-friction, metallo-ceramic protective coatings. The nanocrystalline and multiphase structures reduce internal stress and enhance coating toughness. Due to the grading, the coatings will have metallic adherence to metal substrates, but posses ceramic-like wear resistance at the surface. The Phase I project demonstrated the fabrication of functionally graded, Ti/TiN/TiBN nanocrystalline multiphase ( $\mathrm{TiN}, \mathrm{TiB}_{2}$ and $\mathrm{BN}$ ) coatings with a hardness of 42 Gpa. The graded structure provided a gradual transition from metallic to covalent bonding through the thickness of the film, thereby enhancing adhesion. Wear resistance evaluation (pin-on-disk) for 5 million cycles ( 25 days) at 1 Gpa contact stress showed no wear, indicating the potential for the coating to extend the lifetime of substrate materials by several orders of magnitude. The Phase II project will fabricate and optimize nanocrystalline multiphase (Ti-B$\mathrm{C}-\mathrm{N}$ ) graded layer structures. The ratios between the various film constituents (TiN, TiB, $\mathrm{B}_{4} \mathrm{C}, \mathrm{BN}$ ) will be precisely controlled and related to adhesion, internal stress, hardness, and wear resistance. The coating will be deposited on earth drilling components and will be evaluated for incorporation into drilling products.

Commercial Applications and Other Benefits as described by the awardee: These coatings will find a wide market, particularly as protective coatings for engine components (turbine blades), orthopedic prostheses, other medical devices, and drilling equipment. A major supplier of earth drilling equipment (with a market share of about $\$ 1$ billion per year) will participate as a scientific and commercial partner in Phases II and III.

\section{0}

Large Area Filtered Arc Deposition of Carbon and Boron Based Hard Coatings--UES, Inc., 4401 Dayton-Xenia Road, Dayton, $\mathrm{OH}$ 45432-1805; 937-426-6900

Dr. Rabi S. Bhattacharya, Principal Investigator Mr. Francis F. Williams, Jr., Business Official DOE Grant No. DE-FG02-99ER82911

Amount: \$749,934

Hard, thin, wear resistant coatings can play a vital role in many engineering applications. Many such coatings have been developed over the years in the laboratory by using various plasma-based techniques. However, some of these techniques are not easily scalable for applications on actual engineering components. This project will utilize a large area filtered arc deposition (LAFAD) technique to develop carbon and boron based hard coatings for engineering applications. The filtered arc technique allows deposition of droplet-free smooth coatings by deflecting the plasma flow in a rectangular electromagnetic filter. The advanced filter design utilized in this equipment provide large area deposition ranging from about $250 \mathrm{~mm}$ in width to heights on the order of $300 \mathrm{~mm}$ to $2 \mathrm{~m}$ or more. In Phase $\mathbb{I}$, a number of coatings containing boron and carbon were developed that showed hardness in the range 40 to $65 \mathrm{GPa}$. The boron containing coatings were $\mathrm{TiB}_{2}$ and $\mathrm{TiB}_{\mathrm{x}} \mathrm{N}_{\mathrm{y}}$, whereas the carbon 
containing coatings were diamond like carbon and nanocrystalline $\mathrm{TiC} / \mathrm{C}$. The Phase II project will: (1) continue the development initiated in Phase I through optimization of the coating process for consistent quality with respect to hardness, adhesion, stress and wear resistance, (2) evaluate for machining of steel and aluminum-silicon alloys, (3) evaluate for corrosion resistance against molten aluminum for die casting dies.
Commercial Applications and Other Benefits as described by the awardee: The new technology will significantly improve industrial productivity in machining and die casting, reduce the environmental costs of disposal and fossil energy usage, and provide advanced deposition and equipment technology for commercial opportunities in applications such as biomedicine and electronics.

\section{MATERIALS JOINING}

\section{1}

\author{
Hermetic Reaction Bonded Silicon Carbide \\ Tube Joints with Microwave Heating--FM \\ Technologies, Inc., 10529-B Braddock Road, \\ Fairfax, VA 22032-2236; 703-425-5111 \\ Dr. Ralph W. Bruce, Principal Investigator \\ Dr. Frederick M. Mako, Business Official \\ DOE Grant No. DE-FG02-99ER82922 \\ Amount: $\$ 750,000$
}

Metal alloy tubes in radiant furnaces, widely used for heat treating of steel, operate in severe environments (for example, temperatures in excess of 1000 degrees Centigrade) and require replacement every 1-2 years. Expenses associated with replacing these tubes (including both downtime and the lost of the tubes themselves) are a significant portion of the life cycle cost of heat treating furnaces. Reaction bonded silicon carbide tubes last 2-3 times longer than metal tubes. However, the required 10-15 foot lengths are available at a competitive cost only as straight tubes, whereas 80 percent of radiant tubes are $\mathrm{U}, \mathrm{W}$, or trident shaped. This project will join long straight lengths by forming a silicon carbide-based interlayer in-situ with microwave heating. In Phase I, methods and materials were devised to join short sections of $\mathrm{SiC}$ tubes using a proprietary slurry and microwave heating. The resultant tubes were hermetic with the joint strength equivalent to that of the base material. The Phase II project will join prototype length silicon carbide tube sections using liquid infiltration and pyrolysis of preceramic polymer slurries with microwave heating. Long, commercially usable tubes will then be joined and evaluated for industrial applications.

Commercial Applications and other benefits as described by the awardee: 100 billion cubic feet of natural gas is consumed each year in approximately 25,000 heat treating furnaces that use primarily metal radiant burner tubes. Replacement with ceramic tubes would drastically reduce or eliminate tube replacement cost and enable savings of almost $\$ 200,000$ per furnace per year through increased efficiency of higher temperature operation. 


\section{SUPERCONDUCTIVITY MATERIALS FOR ELECTRIC POWER}

72

\author{
Meter Length YBCO Coated Conductor \\ Development--American Superconductor Cor- \\ poration, Two Technology Drive, Westborough, \\ MA $01581 ; 508-836-4200$ \\ Dr. Cornelius L. H. Thieme, Principal \\ Investigator \\ Dr. Tom Rosa, Business Official \\ DOE Grant No. DE-FG02-99ER82740 \\ Amount: \$750,000
}

Devices such as power transmission cables, motors and generators can be made very compact when run under cryogenic conditions. For further enhancements in power densities it is highly desirable to use High Temperature Superconductors with a greatly enhanced critical current density $\left(\mathrm{J}_{\mathrm{c}}\right.$ at $50-77 \mathrm{~K}$ and magnetic fields of up to 3T) which would allow the operation at much higher temperatures. Production cost of these superconductors needs to be substantially reduced to increase their commercial appeal. The $\mathrm{Yba}_{2} \mathrm{Cu}_{3} \mathrm{O}_{\times}(\mathrm{YBCO})$ coated conductor offers satisfactory performance at high magnetic fields and high temperatures at a greatly reduced cost. This project will produce long lengths of very tiny, non-magnetic substrates with excellent control over substrate dimensions and texture quality, which will be used to manufacture $20 \mathrm{~m}$ long YBCO coated conductor with overall critical current densities of $40,000 \mathrm{~A} / \mathrm{cm}^{2}$ at $77 \mathrm{~K}$. The Phase I project resulted in the development and production of thin, well texture non-magnetic foils. Full buffer stacks (ceria/YSZ/ceria) were successfully deposited, with excellent texture. Solution-based YBCO was successfully deposited and reacted on buffered $\mathrm{Ni}$ substrates with high critical currents. The Phase II project will produce long lengths of thin, non magnetic substrates with excellent control over substrate dimensions and texture quality, which will be used to manufacture $20 \mathrm{~m}$ long YBCO Coated Conductors, with overall critical current densities of $40,000 \mathrm{~A} / \mathrm{cm}^{2}$ at $77 \mathrm{~K}$, self field, and 15,000 $\mathrm{A} / \mathrm{cm}^{2}$ at $50 \mathrm{~K}$ and $3 \mathrm{~T}$.

Commercial Applications and Other Benefits as described by the awardee: The commercial applications of these new YBCO coated conductors are in power transmission cables, motors, generators, transformers, and in magnets for energy storage, magnetic separation, and current limiters. Typically these applications are now addressed by Bi-2223 superconductors. The superior performance of YBCO coated conductor will enable many of these applications to run at higher temperatures and its lower production cost will greatly increase their commercial appeal.

\section{NEUTRON INSTRUMENTATION}

73

An Advanced Cold Moderator Using Solid Methane Pellets--Cryogenic Applications F, Inc., 450 Bacon Springs Lane, Clinton, TN 37716-5311; 865-435-5433

Dr. Christopher A. Foster, Principal Investigator

Dr. Christopher A. Foster, Business Official

DOE Grant No. DE-FG02-99ER82772

Amount: \$749,812
Existing and planned DOE neutron sources, such as the Intense Pulse Neutron Source (IPNS) and the Spallation Neutron Source (SNS), generate pulses of cold neutrons for materials research. The usefulness of such facilities depends on the efficiency of the components that convert the fast neutrons to a cold neutron beam. Although the existing solid methane moderator at IPNS has high efficiency, the design cannot be scaled to the high power levels planned for the SNS. An 
alternative cold moderator concept, which uses solid methane pellets and was proposed in 1988, would be capable of high power operation; however, the technology for producing and transporting the solid pellets have not yet been developed. This project will apply practical pellet fabrication and transport technologies, previously developed for the DOE fusion program, to the production of methane pellets. Phase I demonstrated the production of high quality frozen methane and ammonia pellets. Tests showed that the pellets could be transported into a small scale moderator cell, and refrigerated to temperatures below $20 \mathrm{~K}$. The ammonia pellets were shown to fill the cell with a packing density of 60 percent. Phase II will develop a methane and ammonia pellet fabrication and transport system with the capability of periodically filling a full size cold moderator cell with pellets. The pellets will be refrigerated to below 20K. A method of rapidly replacing the pellets in the moderator cell, which was tested in the Phase I project, will also be demonstrated with the full scale system.

Commercial Applications and Other Benefits as described by the awardee: The cold moderator should enhance the operation of high power spallation neutron sources, which are used for materials and biological science research, by doubling the output of cold neutrons. The technologies developed will also be useful for the production of other cryogenic pellets, such as dry ice, used commercially.

\section{4}

Three-Dimensional Si Imaging Array for Cold Neutrons--IntraSpec, Inc., P.O. Box 4579, Oak Ridge, TN 37830-4579; 423-483-1859

Mr. John Walter, Principal Investigator

Mr. John Walter, Business Official

DOE Grant No. DE-FG02-99ER82807

Amount: $\$ 750,000$
Cold neutron scattering is rapidly gaining importance as a tool for studying structure in materials. Neutron detection arrays are used with such neutron scattering experiments to reduce counting times and increase accuracy. With the advent of stronger sources of cold neutrons, there is a need for much higher response speeds in these arrays. Existing two dimensional wire arrays are limited to about 40,000 counts $/ \mathrm{s}$ total with $10 \%$ dead time and are handicapped by ghost images resulting from pileup problems. This project will develop a 100 pixel sub-array which provides high rate detection, event time, and efficient data collection for the angular distribution of low-energy neutrons scattered during structure studies. Furthermore, the project will demonstrate that these sub-arrays can be combined in a relatively seamless manner to minimize dead regions between them. The Phase I project demonstrated the ability of a small Si pixel detector array to accommodate count rates well in excess of $10^{5}$ counts/s per pixel, as well as the capability to do the fast timing required for simultaneous neutron time-of-flight analysis. A conceptual design was developed for a $10^{4}$ pixel array (composed of 100 pixel sub-arrays, each with parallel processing), a 100 pixel monolithic detector array, and an ASIC parallel signal conditioning array. The Phase II project will design and produce a few neutron imaging sub-arrays with good efficiency and fast timing capability; demonstrate timing and efficiency; and show that adjacent sub-arrays can be combined with minimal dead zone between sub-arrays.

Commercial Applications and Other Benefits as described by the awardee: Instruments for studying long range structural order in materials (by detecting scattered cold neutrons) should benefit by the ability to handle very high rates without the usual problems of pile-up induced ghost images. The technology developed will also prove useful for other applications such as particle physics arrays and $\mathrm{x}$-ray imaging. 
A High-Pressure ${ }^{3} \mathrm{He}$ Neutron Spin Filter-Science Research Laboratory, Inc., 15 Ward Street, Somerville, MA 02143-4228: 617-547-1122

Dr. Jonathan Richardson, Principal Investigator

Dr. Jonah Jacob, Business Official

DOE Grant No. DE-FG02-99ER82878

Amount: \$749,942

Polarized neutron scattering (including small angle reflectometry and refraction) has been demonstrated to be an invaluable tool in the characterization of magnetic and non-magnetic materials. Current neutron polarization methods are limited to well-collimated, low energy beams, precluding their use as spin analyzers in most cases. To overcome these limits, this project will develop a reliable apparatus, utilizing a dense polarized gaseous ${ }^{3} \mathrm{He}$ spin filter, for polarizing ${ }^{3} \mathrm{He}$ with adequate throughput to supply one or more neutron scattering facilities. The system will be able to produce 1 liter-atmosphere of ${ }^{3} \mathrm{He}$ at $>50 \%$ in 1 hour. The application of this system to neutron spin analysis also will be demonstrated. The Phase I project improved an existing polarization apparatus, leading to participation in a polarized neutron reflectometry experiment at the Argonne National Laboratory Intense Pulsed Neutron Source (IPNS). Also, a novel high-power laser was demonstrated and will be used in the improved Phase II polarizer. The Phase II project will address the current deficiencies in the design of the polarizer, develop a next-generation laser, and develop polarizer cells that should offer simpler construction and improved ${ }^{3} \mathrm{He}$ polarization lifetime. Further polarized-neutron measurements are planned at the IPNS.

Commercial Applications and Other Benefits as described by the awardee: There are over nine neutron scattering centers in the US and Canada and over 22 abroad. Currently, plans exist to build a new $\$ 1.36 \mathrm{~B}$ scattering facility in the U.S. called the Spallation Neutron Source (SNS). The technology developed in this project can supply polarization equipment to both existing and future facilities. In addition, polarized ${ }^{3} \mathrm{He}$ can be used for medical imaging and characterization of material microstructure.

\section{ALTERNATIVE SYNTHETIC PATHWAYS FOR ENERGY EFFICIENT AND ECONOMIC INDUSTRIAL CHEMICAL MANUFACTURE}

76

\author{
Novel Catalyst for $\mathrm{CH}_{4}-\mathrm{CO}$ Conversion-- \\ CeraMem Corporation, 12 Clematis. Avenue, \\ Waltham, MA 02453; 781-899-4495 \\ Dr. Michael C.J. Bradford, Principal Investigator \\ Dr. Robert L. Goldsmith, Business Official \\ DOE Grant No. DE-FG02-99ER82762 \\ Amount: $\$ 750,000$
}

In current commercial practice, natural gas is converted to higher molecular weight hydrocarbons through an indirect route in which the natural gas is first converted to synthesis gas (a mixture of hydrogen and carbon monoxide) and subsequently converted to liquids by available technology such as the Fischer-Tropsch process. Considerable economic benefits would accrue if new catalyst and process technologies could directly convert natural gas to liquids. This project will develop a novel catalyst for the direct conversion of methane to heavy aromatics (such as benzene, xylene, and naphthalene), light alkanes and olefins. The process will be suitable for fixed bed reactor technology and is thus capable of being quickly scaled up to commercial capacity. The Phase I project demonstrated the technical feasibility of the proposed process. Active catalysts were prepared, characterized and tested for $\mathrm{CH}_{4}$ dehydro-aromatization. In addition, several multifunctional catalysts were prepared, characterized, and shown to promote 
aromatic formation during $\mathrm{CH}_{4}-\mathrm{CO}$ conversion. Phase II will optimize the catalyst composition and process conditions for $\mathrm{CH}_{4}$ conversion over the novel catalysts developed during Phase 1 . Both experiments and concomitant modeling (of thermodynamics, kinetics, transport phenomena, and process economics) will be undertaken to achieve this objective.
Commercial Applications and Other Benefits as described by the awardee: The technology could significantly increase the utilization of domestic and remote natural gas reserves because it directly converts natural gas to higher molecular weight products of substantially higher market value.

\section{REACTIVE SEPARATIONS}

77

Membrane Reactor Designs for the Production of Olefins--Membrane Technology and Research, Inc., 1360 Willow Road, Suite 103, Menlo Park, CA 94025-1516; 650-328-2228

Dr. J.G. Wiljmams, Principal Investigator

Mr. E.G. Weiss, Business Official

DOE Grant No. DE-FG03-99ER82827

Amount: $\$ 750,000$

Olefins are key feedstocks for the chemical industry. Ten billion lb/year of olefins are made by dehydrogenation of paraffins. Because the equilibrium conversion is low, a large product separation stop is required to separate and recycle unreacted paraffin. Membrane reactors have been shown to improve the equilibrium conversion of dehydrogenation reactions, but a commercial scale reactor has not been developed due to the lack of suitable membrane modules and a process design. This project will develop a membrane reactor process to increase the conversion and reduce the size of the product separation step. The Phase I project devised and modeled a new reactor/membrane separation system on a computer process simulator. Then membranes with the required selectivity were produced. The Phase II project will scale up to large membrane modules and perform tests in the laboratory and at a cooperating field site. Catalyst and reactor studies will be performed to help develop an optimized process design for economic feasibility.
Commercial Applications and Other Benefits as described by the awardee Improved, lower cost olefin production technology should provide significant cost savings for this key feedstock and positively impact the entire chemical industry significantly.

\section{8}

Sonic Assisted Membrane Processing--Montec Associates, Inc., P.O. Box 4182, Butte, MT 59702-4182; 406-494-5555

Mr. Steven F. McGrath, Principal Investigator

Mr. Lawrence C. Farrar, Business Official

DOE Grant No. DE-FG03-99ER82836

Amount: $\$ 750,000$

The economical conversion of many commodity production lines to environmentally friendly biotechnology processes is limited by the downstream operations of separating product from biocatalyst, rather than by the complex technology associated with the directed evolution of enzymes. Although membrane filtration with micro-sized pores has become the separation technology of choice for the biotechnology industry, the protein solutions have a proclivity to foul the membranes during filtration. None of the techniques commonly used to improve ultrafilter performance, such as high cross flow velocity, can be used with proteins. New technology is needed to improve the performance of membrane separations to allow the existing capabilities of biotechnology to evolve into economical processes. This project will address 
the membrane-fouling problem by coupling filtration technology with a mechanically driven low frequency acoustic resonator. The acoustic pulses from the resonator impinging on the membrane create a micro turbulence near the face of the membrane that assists in keeping the flux of permeate from falling due to the buildup of a resistive gel layer. Phase I demonstrated the enhancement of permeate flux through a 1,0,000 molecular weight cut off (MWCO) membrane from solutions with 1 to 5 percent protein (as pepsin). Enhancements were most pronounced for conditions with no cross-flow velocity. These are precisely the conditions in which a free enzyme bioreactor would need to function because of the detrimental effect of cross flow shear on proteins. The Phase II project will optimize the design of a membrane bioreactor for use in specific industrial processes. It will further demonstrate that enzymes proposed for use in these processes are not degraded in the sonic environment. A pilot scale modulator bioreactor will be fabricated and mobilized for continuous testing of the new enzymatic based production.

Commercial Applications and Other Benefits as described by the awardee: In addition to myriad applications in biotechnology, which include traditional fermentation processes, this acoustically enhanced membrane filtration technology should have broad application in industrial separations where membrane usage is predominantly limited by the fouling problem.

Food processing, chemical manufacturing, municipal water treatment, and waste treatment are but a few of the markets that would readily accept a simple new technology that improves membrane performance by a factor of 5 to 10 .

\section{9}

Economic and Self-Sustaining Production of Saleable Products from Waste Anions Using Phase-Transfer Catalysis--PTC Value Recovery, 900 Briggs Road, Suite 130, Mt

Laurel, NJ 08054-4114; 856-642-2080

Dr. Marc Halpern, Principal Investigator Mr. Peter J. Joyce, Business Official 46
DOE Grant No. DE-FG02-99ER82864

Amount: $\$ 723,700$

Many chemical byproducts, which could be converted into useful commercial products, are generated as waste in hundreds of large volume commercial industrial processes. When these byproducts are organic (e.g. phenol derivatives), significant quantities of waste, oftentimes hazardous, pollute the environment and eventually are converted into greenhouse gas $\left(\mathrm{CO}_{2}\right)$ by incineration or biological treatment. In addition, this waste represents significant cost and lost opportunity because it represents inefficient use of raw materials. This project will use Phase-Transfer Catalysis (PTC) technology to produce useful and saleable products from wasted byproducts -- just as it does from hundreds of purchased chemicals in hundreds of commercial processes in a cost efficient manner. In the presence of an appropriate phase transfer catalyst and appropriate conditions, the targeted byproduct species in an aqueous or organic stream will be reacted with a complementary substrate (either nucleophilic or electrophilic, depending on the nature of the byproduct) to make a desirable and saleable product. The Phase I project provided the proof-of-concept for recovering and reacting the commercially important family of high volume hazardous waste and byproduct streams which contain phenol and phenol derivatives. The phenol and phenol derivatives were extracted from low concentration $(\sim 1 w t \%$, ) aqueous streams and successfully reached with a variety of useful electrophiles to produce useful products. Phase II will explore many more major applications that should realize the benefits of PTC in order to advance the technology into more diverse chemistries and industries. In addition, the reaction engineering of these systems will be fully developed and a pilot plant demonstration will be achieved.

Commercial Applications and Other Benefits as described by the awardee: This technology should convert large volumes of phenolic waste and byproducts into useful products such as monomers, and will convert other large volume 
nucleophilic and electrophilic byproducts into saleable products. The technology should also reduce the production of greenhouse gases and reduce the energy load by reducing consumption of natural resources.

\section{INNOVATIVE R\&D FOR ADVANCED, LOW-EMISSION, ELECTRIC AND HYBRID VEHICLE TECHNOLOGIES}

\section{0}

\author{
Flame Retardant Electrolytes for Li-Ion \\ Batteries--EIC Laboratories, Inc., 111 Downey \\ Street, Norwood, MA 02062-2612; \\ 781-769-9450 \\ Dr. Dhamasena Peramunage, Principal \\ Investigator \\ Dr. A.C. Makrides, Business Official \\ DOE Grant No. DE-FG02-99ER82781 \\ Amount: \$747,684
}

The batteries of electric or hybrid electric automobiles may be damaged in an accident. Therefore, for maximum safety, these batteries should contain flame retardant or non flammable electrolytes. This project will develop flame retardant electrolytes for $\mathrm{Li}$-ion batteries offering significantly improved safety in terms of low flammability, electrochemical performance comparable to existing state of the art electrolytes, and the ability to be easily incorporated into existing Li-ion battery technology. The Phase I project demonstrated the feasibility of combining, in the same electrolyte, highly flame retardant behavior and compatibility with Li-ion cell anodes and cathodes. This was achieved by adding commercially available and newly synthesized flame retardant solvents to presently used carbonate solvent blends. The Phase II project will synthesize several new classes of fire retardants and investigate their usefulness in high voltage $\mathrm{Li}$-ion cells. The most promising will be demonstrated in complete $\mathrm{Li}$-ion cells.

Commercial Applications and Other Benefits as described by the awardee: Flame retardant electrolytes should greatly increase the safety of
Li-ion batteries, especially in applications where physical damage to the battery may occur. This includes batteries for electric or hybrid electric vehicles, boats, airplanes, and portable tools, as well as portable electronic devices that are in close proximity to people, such as laptop computers, telephones, and infrared goggles.

\section{1}

Nonflammable Lithium-Ion Battery

Electrolytes--TechDrive, Inc., 17 W 695

Butterfield Road, Suite A, Oakbrook Terrace, IL 60181; 630-910-3729

Dr. Robert Filler, Principal Investigator

Dr. Robert Filler, Business Official

DOE Grant No. DE-FG02-99ER82905

Amount: $\$ 750,000$

Small lithium-ion batteries are now employed in power cellular phones, computers, and cameras. However, before full size batteries can be mass produced for use in electric vehicles, safetyrelated issues, including flammability of electrolytes, must be addressed. This project will develop significantly improved electrolyte systems that exhibit a high degree of nonflammability and higher flash points, while retaining the current lithium ion battery technology. The Phase I project prepared novel cost-effective flame-retarding additives to the electrolytes for lithium-ion batteries. The materials were found to exhibit very good to excellent thermal and electrochemical stabilities. The Phase II project will synthesize the best flame-retardant additive, optimize it for bulk production, fully characterize the material (including an evaluation of its ability to 
self-extinguish flammable battery electrolytes), and test it in full size batteries.

Commercial Applications and Other Benefits as described by the awardee: The availability of safer lithium-ion batteries incorporating self- extinguishing flammable electrolytes should have a wide range of applications in consumer products (cell phones, computers, cameras), automotive products (including electric vehicles) as well as in weapons systems for the military.

\section{COMPUTATIONAL GEOSCIENCES}

\section{2}

\author{
A Geometry-Based Interactive Pre- and \\ Post-Processor for Reservoir Simulation and \\ Visualization--Thunderhead Engineering \\ Consultant, Inc. (Joint Venture), 1006 Polyntz \\ Ave, Manhattan, KS 665025459; \\ 785-587-8492 \\ Dr. Daniel Swenson, Principal Investigator \\ Dr. Daniel Swenson, Business Official \\ DOE Grant No. DE-FG03-99ER82908 \\ Amount: $\$ 545,320$
}

The utility of current geothermal reservoir simulators is difficult because they were developed before interactive user interfaces were common. Although the simulators can solve complex problems, it takes a significant investment to become proficient in simulator use. New computational capabilities and software design approaches present an opportunity to simplify and enhance cur-rent methods used to create and view reservoir simulations. This project will de-velop an interactive, crossplatform, pre- and post-processor that can be used to create reservoir models and display simulation results through time. The program will help users create the input files needed for the existing TOUGH2, TETRAD, and STAR simulation programs. After the calculations are complete, the computer program will allow the user to rapidly access and visualize the results. In Phase I, an operational program prototype was developed to demonstrate the viability of using a geometric model database to support multiple reservoir simulation codes. The threedimensional display allows the user to interact with the model during creation. A subset of features was implemented to generate input files for simulation with the TOUGH2 and TETRAD codes. Phase II will result in a commercial preand post-processor computer program for use with the TOUGH-2, TETRAD, and STAR reservoir simulators. The Phase II project will add all the features needed for comprehensive reservoir simulation, including a high level geometric representation to support interactive visualization of the data.

Commercial Applications and Other Benefits as described by the awardee: Computer simulation of reservoirs and environmental remediation is increasingly important to the Nation, both with regard to energy and the environment. This software should allow existing simulators to be accessible to a larger user base. 


\section{HIGH PERFORMANCE NETWORKS AND APPLICATIONS}

83

Al, An ESNET Differentiated Services Allocation System--Aldea Communication, Inc., 7720 El Camino Real, \#425, Carlsbad, CA 92009-8508; 760-510-8406

Ms. Susan Estrada, Principal Investigator

Ms. Susan Estrada, Business Official

DOE Grant No. DE-FG03-99ER82831

Amount: \$375,000

A management policy is needed for allocating Internet Protocol Quality of Service (IP QoS) among the users of DOE's ESnet (Energy Sciences Network) and for deploying that policy into bits per-second. This project will develop a software program, structured as a policy management tool, to allow less sophisticated network personnel to intelligently configure their IP QoS using human policies. Phase I demonstrated the concept of translating human policy into QoS policies. Design information was gathered from the literature as well as from QoS researchers and standards bodies. The findings narrowed the design of the policy management tool. The Phase II project will further develop software to transform human policy information into data that can be formatted into network-ready policy information. The software structure will include a wizard feature to simplify the translation of human policy to QoS-speak, a relational database to translate that policy into accurate and useful configuration files for improved network operation, an input interface component, and an output interface component.

Commercial Applications and Other Benefits as described by the awardee: The target market for this software product is enterprise internets and the Internet. The software should provide a simple way to configure various network devices for QoS and to validate what was deployed. Router, switch, and policy server vendors should seek such tools to enhance their IP QoS product lines.
84

Holographic Memory Module with Ultra-High

Capacity and Throughput--MetroLaser, Inc., 18010 Skypark Circle, Suite 100, Irvine, CA 92614-6428; 949-553-0688

Dr. James E. Trolinger, Principal Investigator

Dr. Cecil Hess, Business Official

DOE Grant No. DE-FG03-99ER82833

Amount: $\$ 749,994$

High capacity, high transfer rate, random access memory systems are needed to archive and distribute the tremendous volume of digital information being generated for such applications as human genome mapping and online libraries. The development of multi-gigabit-per-second networks underscores the need for nextgeneration archival memory systems. This project will develop a novel holographic memory that has ultra-high data storage capacity in excess of $10^{15}$ bits storage and throughput of 1 Gbit/sec. The proposed method is based on a revolutionary concept in holographic multiplexing -- the technique that leads to a compact and robust architecture. Stored data will be recalled only with a decoder-key that is identical to that used at the recording stage. In Phase I, the theoretical analysis was conducted, and experimental tests were performed to validate key aspects of the data storage module. The secure nature of the encoding method was inspected and the performance of a full-scale system was estimated. Phase II will develop a novel holographic memory module with ultrahigh data storage capacity and throughput. System design will be optimized through analysis and experiments, a memory system prototype will be built, its performance will be measured, and highly secure data access will be demonstrated.

Commercial Applications and Other Benefits as described by the awardee: The memory system should be well suited for high security applications, such as in government, because of 
how the data is encrypted. In the consumer market, the high-speed access offers a significant improvement over magnetic media for storing the operating system and application software for personal computers, libraries of films or books, and large databases of navigational information.

\section{5}

Plug-and-Play Phase Conjugate Quantum Cryptography for Secure Private and Public Key Distribution--Physical Optics Corporation, 20600 Gramercy Place, Building 100, Torrance, CA 90501-1821; 310-320-3088

Dr. Andrew Kostrzewski, Principal Investigator

Mr. Gordon Drew, Business Official

DOE Grant No. DE-FG03-99ER82869

Amount: \$749,998

The U.S. Department of Energy (DOE) is seeking new technologies that can improve communication security and reduce the spread of protected information. Existing products have intrinsic limitations that either prevent full security or make them impractical in real life applications. This project will utilize quantum cryptography technology to improve the security and privacy of U.S. government communications and commercial electronic trade. A unique Phase Conjugate Quantum Cryptography (PCQC) system will be developed to ensure truly secure communications by means of phase conjugate optics. Phase I demonstrated the feasibility of producing all critical components, and developed a simulation of the entire system. A novel optical phase conjugate mirror was developed and integrated it into a stable phase conjugate interferometric system. The results indicated that the PCQC technology is technically viable, with strong competitive advantages over existing systems. Phase II will focus on developing a full prototype of the PCQC system and on implementing it on an existing local area network. This will include optimizing the optical designs and components of the PCQC system, developing electronic processors and software, and implementing optimum encoding procedures. The system will be evaluated by LANL and by commercial firms.
Commercial Applications and Other Benefits as described by the awardee: PCQC products should be economical, user-friendly, and compatible with commercial local area networks. Commercial applications are in LAN security and private network encryption. Other applications are in e-commerce for secure financial data exchange, video encryption, and medical data encryption. In addition, PCQC technology could be used in adaptive interferometers and adaptive optical systems for phase correction in astronomy and in surveillance activities, by law enforcement, the U.S. military, and NASA.

\section{6}

Low Cost 10Gb/s Optical Links for Gigabit Networks--Princeton Electronic Systems, Inc., P.O. Box 8627, Princeton, NJ 08543-8627; 609-799-5414

Dr. Reza Saedi, Principal Investigator

Dr. Chuni Ghosh, Business Official DOE Grant No. DE-FG02-99ER82870

Amount: $\$ 750,000$

Affordable gigabit networks are necessary for communication at high data rates in the business and scientific world. To make affordable gigabit networks a reality, low cost multi-gigabit links are essential. This project will develop front ends for low cost multi-gigabit networks by developing $10 \mathrm{~Gb} / \mathrm{s}$ transmitter and receiver modules for multi-gigabit fiber optical networks. Different designs will be used to address the short and long reach application requirements. The Phase I project developed, designed and tested a prototype transmitter and receiver pair operating at $10 \mathrm{~Gb} / \mathrm{s}$. The transmitter and receiver meet all operating design requirements and specifications. The Phase II project will further improve the performance and reduce the production cost of the transmitter and receiver modules using vertical cavity surface emitting laser based technologies. The short reach transmitter will be based on low cost direct modulation of laser, whereas an integrated laser and modulator will be used in the long reach transmitter module. 
Commercial Applications and Other Benefits as described by the awardee: There is tremendous demand for data bandwidth for every conceivable scientific and commercial applications. The gigabit fiber optical links for high speed data transmission for local data networks as well as for the gateways for external communications should have a huge market potential.

\section{ADVANCED SENSORS AND DATA ANALYSIS TECHNIQUES FOR NATIONAL SECURITY APPLICATIONS}

87

\author{
Chemosensor Array for Detecting the \\ Proliferation of Weapons of Mass Destruction- \\ -Intelligent Optical Systems, Inc., 2520 West \\ 237th Street, Torrance, CA 90505-5217: \\ 310-530-7130 \\ Dr. Kisholoy Goswami, Principal Investigator \\ Mr. Robert Lieberman, Business Official \\ DOE Grant No. DE-FG03-99ER82805 \\ Amount: \$749,998
}

The DOE's Nonproliferation and National Security (NN) Program needs new and innovative sensor systems that can be used for counter-proliferation treaty monitoring, counterterrorism, and the detection of smuggled nuclear and chemo/bio warfare materials. Optical evanescent wave chemical sensors are being considered by NN for detecting and characterizing signature molecules related to weapons of mass destruction. This project will develop innovative chemosensor arrays and utilize neural net signal processing algorithms to substantially improve the performance of these sensors. Phase I developed three key innovations: (a) a reversible optical sensor demonstrating $\mathrm{ppb}$ level sensitivity to kerosene and tributyl phosphate (materials associated with processing nuclear materials), (b) sensing materials having the potential for detecting target species selectively from a mixture, and (c) an approach that allows the construction of handheld instruments for use by minimally skilled personnel. These innovations will allow sensitive and selective detection of undesired activities by rogue nations, and by terrorist groups and individuals. Phase II will develop a prototype sensor system, and components will be optimized for low parts-per-billion detection of a variety of organic vapors related to nuclear processing, chemical warfare agents, and explosives. The prototype will be field-tested for performance verification.

Commercial Applications and Other Benefits as described by the awardee: The proposed product will have a direct impact on national security and environmental monitoring. Spin-off products should emerge that can be used in air quality monitoring, gas detection, process control industries, and law enforcement.

\section{8}
A New Microfluidic System for Detection of Bacterial Agents on Contaminated Surfaces-- Lynntech, Inc., 7610 Eastmark Drive, Suite 202, College Station, TX 77840-4023; 979-693-0017
Dr. Dalibor Hodko, Principal Investigator
Dr. Oliver J. Murphy, Business Official
DOE Grant No. DE-FG03-99ER82816
Amount: \$749,909

Recent developments of biological agents as weapons have paralleled advances in basic microbiology and present a constant threat worldwide. The sensitivity and speed of methods for their detection need to be constantly improved to provide timely and safe response in the case of their deployment. New detectors are urgently needed that will combine sampling of contaminated objects with the identification of a broad spectrum of biological agents. This project will develop a non-optical detection method consisting of a practical microfluidic 
device which integrates sampling, concentration, and detection of biological agents found on objects or people. The system will utilize a polymerase chain reaction (PCR), DNA amplicons specific of each pathogen, to allow further miniaturization, ruggedness, and decreased measurement time. Phase I fully demonstrated the feasibility of detecting surrogate biological agents using a new amplified electrochemical detection of DNA amplicons obtained through the PCR chemistry. Detection of single and double stranded DNA of varying size was achieved. Basic design parameters for a microfluidic system were determined, as were ways to further enhance system sensitivity. Phase II will optimize the detection and engineering design parameters of the prototype microfluidic device. Detection of biological agents surrogates will be optimized with respect to the interference of other biological particles on the measurement sensitivity. The proposed method will be tested for the potential to detect pathogens where only one or a few DNA molecules are present in the sample.

Commercial Applications and Other Benefits as described by the awardee: The new method for integrated sampling and detection of biological particles and detecting DNA molecules will find, apart from the application in the biological warfare, a wide range of applications in biomedical and pharmaceutical drug testing, genomic research, and in food, environmental and forensic bacteriological analysis.

\section{9}

Compact Reflector Telescope--Optra, Inc., 461

Boston Street, Topsfield, MA 01983-1290; 978-887-6600

Dr. Michael Hercher, Principal Investigator

Mr. James R. Engel, Business Official

DOE Grant No. DE-FG02-99ER82848

Amount: $\$ 528,110$

A Raman Spectrometer for the remote detection and identification of containments, hazardous materials, forensic evidence, etc., has been developed by a group at the Brookhaven National 52
Laboratory. In order for it to be a valuable field instrument for use by Government agencies and industry, its radiometric efficiency must be significantly improved so that its size and cost can be reduced. This project addresses this problem by: (1) designing a more efficient collection telescope tailored to work at the short stand off ranges for which the system is intended, (2) designing a focusing transmitter telescope to reduce the size of the excitation beam at the target surface, and (3) developing a fiber-optic image transformer to convert the circular image formed by the collection telescope into a slitshaped image to match the entrance pupil of the spectrometer. In Phase I, a prototype fiber-optic image transformer was made that provided a 25 percent to 40 percent increase in the signal at the detector compared to the system currently in use. Phase II will review the design of the new optical system, build the redesigned optical system, integrate it into the Mini-Raman Lidar System at Brookhaven, fully evaluate the performance of the up-graded system, and complete the manufacturing engineering necessary to reduce the cost and weight of the overall system to make it a commercially viable product.

Commercial Applications and Other Benefits as described by the awardee: The commercial applications for a complete Min-Raman Lidar System include remote identification of potentially hazardous spills, stand-off detection and identification of chemical and biological warfare agents, industry process control, quality control in the food-processing industry, and nonintrusive forensic applications.

90

Fast Analysis of Ultraspectral Measurements of Chemical Plumes--Spectral Sciences, Inc., 99 South Bedford Street, \#7, Burlington, MA 01803-5153; 781-273-4770

Dr. Marsha J. Fox, Principal Investigator

Dr. Fritz Bien, Business Official

DOE Grant No. DE-FG02-99ER82889

Amount: $\$ 750,000$ 
The timely detection, identification, and quantification of chemical plumes, using ultraspectral sensors from air- or space-borne platforms is needed for national security applications. For accurate plume analysis, atmospheric correction is critical, especially at low concentrations and temperatures. This project will develop plume detection and quantification algorithms, including atmospheric correction, for integration into a versatile hyperspectral data analysis package for use by: data analysts. In Phase I, a physical model of the plume/atmosphere/ground interaction was mathematically formulated. Ultraspectral scenes containing plumes were simulated, and the detection and quantification concept was demonstrated with atmospheric correction. Improved performance and accurate plume quantification was shown to outperform methods that ignore atmospheric correction. Phase II will develop a software toolkit for the data analyst to perform fast analysis using advanced detection and quantification algorithms, and to accurately model atmosphere and scene parameters. The toolkit will include atmospheric correction and chemical analysis utilities, a scene simulation utility, and a high spectral resolution atmospheric modeling utility.

Commercial Applications and Other Benefits as described by the awardee: Applications include remote sensing for precision agriculture, mineral detection, forest fire characterization, pollution characterization and control, drug interdiction and national security systems.

\section{ADVANCED RESEARCH IN SUPPORT OF THE COMPREHENSIVE NUCLEAR-TEST BAN TREATY}

91

\author{
Seismic Monitoring System Calibration Using \\ Ground Truth Database--Multimax, Inc., 1441 \\ McCormick Drive, Largo, MD 20774-5323; \\ 301-925-8222 \\ Dr. W. Winston Chan, Principal Investigator \\ Dr. W. Winston Chan, Business Official \\ DOE Grant No. DE-FG02-99ER82837 \\ Amount: $\$ 749,558$
}

Precise location and high confidence identification of regional seismic events are the primary objectives of research required for seismic monitoring of the Comprehensive Test Ban Treaty (CTBT). However, calibration of a monitoring system is a major issue in CTBT verification. Due to the lack of ground truth information and uncertainties in regional geological parameters, rapid and accurate identification of suspicious events is currently not feasible. This project will develop a systematic compilation and analysis scheme for ground truth information using sets of geophysical data assembled from China as a test case. In Phase I, a data product consisting of ground-truth information for central China was delivered to Los Alamos National Laboratory for integration with the DOE's knowledge base of ground truth information. For the first time, regional data consisting of seismic arrival phase picks, geological maps, and travel time models have been made available and assembled in a standard format. A tomographic inversion for threedimensional velocity models was developed to allow travel time correction of surface calculations. The Phase II project will expand this effort into providing a comprehensive database for all China. Additionally, digital waveform data from the Chinese Digital Seismic Network will be analyzed along with data from the regional digital seismic networks. Tomographic inversion will be performed to obtain regional three-dimensional velocity models for calculation of travel time correction surfaces.

Commercial Applications and Other Benefits as described by the awardee: The ground truth 
database for China will be very useful to the $\mathrm{DOE}$ and other researchers for monitoring the CTBT. Similar research products could also be used by the Nuclear Regulatory Commission for nuclear reactor site design; the Federal Highway Administration for bridge and highway designs; and engineering firms for earthquake safety fabrication design. International insurance firms could use the product to aid in calculating the insurance risk in China related to earthquakes and other natural disasters. 


\section{APPENDIX A}

\section{Alphabetical List of Awardees}

Advanced Energy Systems, Inc. $43,44,45$

Mr. Anthony Favale, Business Official

27 Industrial Boulevard

Unit E

Medford, NY 11763-2286

631-345-6264

AIL Research, Inc. 11

Dr. Andrew Lowenstein, Business Official

P.O. Box 3662

50 Washington Road

Princeton, NJ 08543-3662

$609=452-2950$

Aldea Communication, Inc. 83

Ms. Susan Estrada, Business Official

7720B El Camino Real

\#425

Carlsbad, CA 92009-8508

760-510-8406

Altex Technologies Corporation

1

Dr. Mehdi Namazian, Business Official

650 Nuttman Street

Suite 114

Santa Clara, CA 95054-2620

408-982-2303

AMAC International, Inc. 46

Ms. Loren Loving, Business Official

Applied Research Center

12050 Jefferson Avenue, Suite 348

Newport News, VA 23606-4323

757-249-3595

American Superconductor Corporation 72

Dr. Tom Rosa, Business Official

Two Technology Drive

Westborough, MA 01581

508-836-4200
Applied Sciences, Inc. 52

Mr. Max L. Lake, Business Official

141 West Xenia Avenue

P.O. Box 579

Cedarville, $\mathrm{OH}$ 45314-0579

937-766-2020

Avyd Devices, Inc. 56,57

Dr. Honnavalli R. Vydyanath, Business Official 2925 College Avenue

Unit A-1

Costa Mesa, CA 92626

714-751-8553

Berkeley Ion Equipment, Inc. 47

Mr. M. Wei, Business Official

3400 De La Cruz Boulevard

Suite V

Santa Clara, CA 95054-2609

408-727-9071

Blasch Precision Ceramics, Inc. 13

Mr. David W. Bobrek, Business Official

580 Broadway

Albany, NY 12204-2802

518-436-1263

Blue Road Research 55

Mr. Eric Udd, Business Official

2555 N.E. 205th Avenue

Fairview, OR 97024-8600

503-667-7772

Calabazas Creek Research 28

Dr. R. Lawrence Ives, Business Official

20937 Comer Drive

Saratoga, CA 95070-3753

408-741-8680 
California Tube Laboratory, Inc.

Mr. Peter S. Campbell, Business Official

125 Aviation Way

Watsonville, CA 95076-2058

831-761-1199

Ceradyne, Inc. 48

Mr. Howard George, Business Official

3169 Red Hill Avenue

Costa Mesa, CA 92626

714-549-0421

CeraMem Corporation $14,17,76$

Dr. Robert L. Goldsmith, Business Official

12 Clematis Avenue

Waltham, MA 02453

781-899-4495

Chesapeake Composites Corporation 67

Dr. Alexander Brown, Business Official

239 Old Churchman's Road

New Castle, DE 19720-1529

302-324-9110

Composite Technology Development, Inc. 31

Dr. Naseem A. Munshi, Business Official

1505 Coal Creek Drive

Lafayette, CO 80026-2782

303-664-0394

Cryogenic Applications F, Inc. 73

Dr. Christopher A. Foster, Business Official

450 Bacon Springs Lane

Clinton, TN 37716-5311

865-435-5433

CyberConnect Corporation 64

Mr. Karl Beckert, Business Official

103 Moulton Road

Storrs, CT 06268

860-429-2666

DAC Vision, Inc. 36

Mr. James W. Drain, Business Official

6390 Rose Lane

Carpinteria, CA 93013

805-684-8307
Diversified Technologies, Inc. $20,30,37,38$

Mr. Michael A. Kempkes, Business Official

35 Wiggins Avenue

Bedford, MA 01730-2314

781-275-9444

EIC Laboratories, Inc. 80

Dr. A.C. Makrides, Business Official

111 Downey Street

Norwood, MA 02062-2612

781-769-9450

Eltron Research, Inc.

Ms. Eileen E. Sammells, Business Official

4600 Airport Boulevard

Boulder, CO 80301-3241

303-530-0236

Energen, Inc. 49

Dr. Chad H. Joshi, Business Official

17 D Sterling Road

Billerica, MA 01862-2518

978-671-5400

FARTECH, Inc 19

Dr. Jin-Soo Kim, Business Official

3146 Bunche Avenue

San Diego, CA 92122-2247

858-455-6655

FM Technologies, Inc. 71

Dr. Frederick M. Mako, Business Official 10529-B Braddock Road

Fairfax, VA 22032-2236

703-425-5111

Genome Informatics Corporation 65

Dr. Ed Uberbacher, Business Official

1020 Commerce Park Drive

Oak Ridge, TN 37830

55697-99-II

423-220-0043 
Hi-Z Technology, Inc.

9

Mr. Norbert Elsner, Business Official

7606 Miramar Road

Suite 7400

San Diego, CA 92126-4202

619-695-6660

Hypres, Inc. 41

Dr. Elie Track, Business Official

175 Clearbrook Road

Elmsford, NY 10523-1109

914-592-1190

HYTEC, Inc. 40

Mr. William O. Miller, Business Official

110 Eastgate Drive, Suite 100

Los Alamos, NM 87544-3304

505-662-0080

Intelligent Optical Systems, Inc. 87

Mr. Robert Lieberman, Business Official

2520 West 237th Street

Torrance, CA 90505-5217

310-530-7130

IntraSpec, Inc. 74

Mr. John Walter, Business Official

P.O. Box 4579

Oak Ridge, TN 37830-4579

423-483-1859

Linear Measurements, Inc. 3

Mr. Robert Hatch, Business Official

4174 Sorrento Valley Boulevard

San Diego, CA 92121-1407

858-535-2172

LMC, Inc. 39

Mr. Lennart J. Lindell, Business Official

333 Dietz Avenue

De Kalb, IL 60115

815-758-3514
Lynntech, Inc. 53,88

Dr. Oliver J. Murphy, Business Official

7610 Eastmark Drive

Suite 202

College Station, TX 77840-4023

979-693-0017

Materials Modification, Inc. 68

Dr. T.S. Sudarshan, Business Official

2929 P-1 Eskridge Road

Fairfax, VA 22031-2213

703-560-1371

Membrane Technology and Research, Inc. 77

Mr. E.G. Weiss, Business Official

1360 Willow Road

Suite 103

Menlo Park, CA 94025-1516

650-328-2228

MER Corporation

Dr. J. C. Withers, Business Official

7960 South Kolb Road

Tucson, AZ 85706

520-574-1980

MetroLaser, Inc. 6,84

Dr. Cecil F. Hess, Business Official

18010 Skypark Circle

Suite 100

Irvine, CA 92614-6428

949-553-0688

Montec Associates, Inc. 78

Mr. Lawrence C. Farrar, Business Official

P.O. Box 4182

55790-99-II

406-494-5555

Multimax, Inc.

Dr. W. Winston Chan, Business Official 1441 McCormick Drive

Largo, MD 20774-5323

301-925-8222 
Nanomaterials Research Corporation 7,58

Dr. Tapesh Yadav, Business Official

2620 Trade Center Avenue

Longmont, $\mathrm{CO}$ 80503-7551

303-702-1672

Omega-P, Inc. 25

Mr. George P. Trahan, Business Official

202008 Yale Station

Suite 100

New Haven, CT 06520

203-789-1164

Optra, Inc. 89

Mr. James R. Engel, Business Official

461 Boston Street

Topsfield, MA 01983-1290

978-887-6600

PEM Technologies, Inc. 62

Dr. Irving Weinberg, Business Official

5611 Roosevelt Street

Bethesda, MD 20817-6739

301-564-0835

Photon Imaging, Inc. 50,63

Dr. Bradley E. Patt, Business Official

19355 Business Center Drive

Suite 8

Northridge, CA 91324-3503

818-709-2468

Physical Optics Corporation $59,66,85$

Mr. Gordon Drew, Business Official

20600 Gramercy Place

Building 100

Torrance, CA 90501-1821

310-320-3088

Physical Sciences, Inc. 61

Dr. Byron David Green, Business Official

20 New England Business Center

Andover, MA 01810-1077

978-689-0003
Plasma Processes, Inc.

Mr. Timothy McKechnie, Business Official

4914-D Moores Mill Road

Huntsville, AL 35811-1558

256-851-7653

Plastronic, Inc., Subsidiary of Eurus Technologies

Inc. 32

Mr. John A. Romans, Business Official

2031 E. Paul Dirac Drive

Innovation Park

Tallahassee, FL 32310

800-574- 1800

Positive Light, Inc.

Mr. Jeremy Weston, Business Official

101 Cooper Court

Los Gatos, CA 95032-7604

408-399-7744

PowerLight Corporation 12

Mr. Thomas L. Dinwoodie, Business Official

2954 San Pablo Avenue

Berkeley, CA 94710

510-540-0550

Precision Combustion, Inc. 15

Mr. Paul M. Donahe, Business Official

410 Sackett Point Road

New Haven, CT 06473-3106

203-787-8614

Princeton Electronic Systems, Inc. 86

Dr. Chuni Ghosh, Business Official

P.O. Box 8627

Princeton, NJ 08543-8627

609-799-5414

PTC Value Recovery 79

Mr. Peter J. Joyce, Business Official

900 Briggs Rd.

Suite 130

Mt. Laurel, NJ 08054-4114

856-642-2080 
Radiation Monitoring Devices, Inc.

Dr. Gerald Entine, Business Official

44 Hunt Street

Watertown, MA 02472-4699

617-926-1167

Science Research Laboratory, Inc. 75

Dr. Jonah Jacob, Business Official

15 Ward Street

Somerville, MA 02143-4228

617-547-1122

Scientific Digital Visions, Inc. 42

Ms. Tracy Langlands, Business Official

2 North Second Street

Suite 1215

San Jose, CA 95113-1303

408-289-8494

Sigma Technologies International, Inc. 4

Dr. Angelo Yializis, Business Official

10960 N. Stallard Place

Tucson, AZ 85737-9527

$520-575-8013$

Silicon Designs, Inc. 5

Mr. John C. Cole, Business Official

1445 NW Mall Street

Issaquah, WA 98027

425-391-8329

Spectral Sciences, Inc. 90

Dr. Fritz Bien, Business Official

99 South Bedford Street

Unit 7

Burlington, MA 01803-5153

781-273-4770

Spinheat Limited 2

Mr. Michael J. Virr, Business Official

1222 Bronson Road

Fairfield, CT 06430-2824

203-259-6101
Spire Corporation. 69

Dr. Richard S. Gregorio, Business Official

One Patriots Park

Bedford, MA 01730-2396

781-275-6000

Starmet Corporation 54

Mr. Donald T. King, Business Official

2229 Main Street

Concord, MA 01742

978-369-5410

Supercon, Inc. 33

Mrs. Elaine Tarkiainen, Business Official

830 Boston Turnpike

Shrewsbury, MA 01545-3301

508-842-0174

Superconducting Systems, Inc.

Dr. Shahin Pourrahimi, Business Official

90 Rumford Avenue

Waltham, MA 02453

781-642-6702

Supergenics 35

Mr. Bruce A. Zeitlin, Business Official

1233 Tree Bay Lane

Sarasota, FL 34242

941-349-0930

TDA Research, Inc. 16

Mr. Michael E. Karpuk, Business Official

12345 West 52nd Avenue

Wheat Ridge, CO 80033-1917

303-940-2301

Tech-X Corporation 27

Dr. John R. Cary, Business Official

5541 Central Avenue

Suite 135

Boulder, CO 80301

303-448-0727 
TechDrive, Inc.

81

Dr. Robert Filler, Business Official

17 W 695 Butterfield Road

Suite A

Oakbrook Terrace, IL 60181

630-910-3729

Thunderhead Engineering Consultants, Inc.

(Joint Venture)

Dr. Daniel Swenson, Business Official

1006 Polyntz Avenue

Manhattan, KS 665025459

785-587-8492

UES, Inc.

Mr. Francis F. Williams, Jr., Business Official

4401 Dayton-Xenia Road

Dayton, $\mathrm{OH}$ 45432-1805

937-426-6900

Unique Mobility, Inc. 10
UTRON, Inc. 23

Mr. Dennis Massey, Business Official

8506 Wellington Road, Suite 200

Manassas, VA 20109-3915

703-369-5552

X-Ray Optical Systems, Inc. 8

Mr. David Usher, Business Official 30 Corporate Circle

Albany, NY 12203

518-464-3334

Yankee Environmental Systems, Inc. 60

Ms. Cynthia A. Cote, Business Official 101 Industrial Boulevard

Turners Falls, MA 01376-1608

413-863-0200

Mr. Donald A. French, Business Official

425 Corporate Circle

Golden, CO 80401-5635

303-278-2002 


\title{
APPENDIX B
}

\section{State List of Awardees}

\author{
Alabama ..........................22 \\ Arizona $. . . . . . . . . . . . . . . . . . . .4,24$ \\ California ................. 1, 3, 6, 9, 12, 19.26, 28, 29, 36, 42, 47, 48, 50, 56, 57, 59, 63, 66, 77, 83, 84, 85, 87 \\ Colorado \\ $7,10,16,18,21,27,31,58$ \\ Connecticut \\ $2,15,25,64$ \\ Delaware \\ 67 \\ Florida \\ 32,35 \\ Illinois \\ 39,81 \\ Kansas \\ 82 \\ Maryland \\ 62,91 \\ Massachusetts \\ $14,17,20,30,33,34,37,38,49,51,54,60,61,69,72,75,76,80,89,90$ \\ Montana \\ 78 \\ New Mexico \\ 40 \\ New Jersey \\ $11,79,86$ \\ New York \\ $8,13,41,43,44,45$ \\ Ohio \\ 52,70 \\ Oregon \\ 55 \\ Tennessee \\ $65,73,74$ \\ Texas \\ 53,88 \\ Virginia \\ $23,46,68,71$ \\ Washington \\ 5
}




\section{APPENDIX C}

\section{Subject Index}

The Subject Index has two parts: DOE Program Subjects and Crosscutting Subjects. The numbers which follow each subject identify the abstracts as they appear in the Table of Contents.

\section{DOE PROGRAM SUBJECTS}

Energy Production

Fossil Energy, 13-18

Fusion Energy Sciences, 19-24

Renewable Energy

Biomass, $1-2$

Geothermal Drilling, 3-5

Photovoltaics and Solar Energy, 11-12

Energy Usage

Electric Vehicles, 9-10, 80-81

Buildings and Industry, 6-8, 76-79

Impact on the Environment

Environmental Management, 50-54

Environmental Research, 55-61

Nonproliferation and National Security, 87-91

High Energy and Nuclear Physics

Accelerator Science and Technology, 25-39, 43-49

Detectors, Instrumentation, and Data Processing, 40-42

Underlying Energy Sciences

Materials, 67-75

Life and Health Sciences, 62-66

Computational Technology, 82-86

\section{CROSSCUTTING SUBJECTS}

Batteries, 80-81

Beams and Beam Diagnostics, 8, 9, 47

Catalysts, Chemistry, Electrochemistry, 15, 16, 76, 79, 87, 90

Data Processing, Networking, Communication, 42, 64, 65, 83-86, 90,91

Electronic Components, 3, 4, 20, 23, 37 
Fuel Cells, 13-14

Genetics, DNA, 64-66

Imaging, 51, 62, 63, 74, 82

Instrumentation, $7,8,50,73-75$

Laser Technology, 26

Materials Development, Processing, 12, 22, 23, 37, 40, 42, 49, 68-76

Membranes, 18, 77

Motors, 9-10

Nanotechnology, 69, 7.0

Optical Components, Fiber Optics, Laser, 51, 74

Pollution Control, 52-55, 81

Scientific Computing, and Simulations, 25, 47, 82, 83

Sensors, 5, 6, 20, 42, 50, 51, 57-59, 75, 87-89

Superconducting and Magnet Technology, Gryogenics, 3.1-36, 44-46

Thin Films and Coatings, 24, 69, 70, 72, 73 